\title{
REPRESENTATION TYPE OF FINITE QUIVER HECKE ALGEBRAS OF TYPE $D_{\ell+1}^{(2)}$
}

\author{
SUSUMU ARIKI AND EUIYONG PARK
}

ABStRACT. We give an Erdmann-Nakano type theorem for the finite quiver Hecke algebras $R^{\Lambda_{0}}(\beta)$ of affine type $D_{\ell+1}^{(2)}$, which tells their representation type. If $R^{\Lambda_{0}}(\beta)$ is not of wild representation type, we may compute its stable Auslander-Reiten quiver.

\section{INTRODUCTION}

This paper is the second of our series of papers on the representation type of finite quiver Hecke algebras. Categorification is popular in various fields of mathematics recently. One purpose of categorification is for a deeper understanding of the objects to be categorified. But there is another purpose: categorification is a method to relate various categories in a systematic way. Categorification of integrable modules over a Kac-Moody Lie algebra is such an example, and in the early 1990's, a categorification of integrable modules over an affine Kac-Moody algebra of type $A_{\ell}^{(1)}$ was found to be useful for studying module categories of finite dimensional Hecke algebras. They were studied by the first author and his collaborators. Then, generalization of this categorification to other Lie types by using cyclotomic quiver Hecke algebras, which are also called cyclotomic Khovanov-Lauda-Rouquier algebras, has attracted the interest of various mathematicians in the 2000's.

In any representation theory, classification of irreducible modules is the starting point. But remaining at that stage is not very interesting, and we must handle nonsemisimple modules. To obtain a result, one studies a special kind of non-semisimple module, and various methods have been developed in each representation theory.

In our context, we are given a new type of self-injective algebra, cyclotomic quiver Hecke algebras, and it is worth pursuing to develop new ways of obtaining results, namely, proof techniques that combine categorification of integrable modules over an affine Kac-Moody algebra with classical techniques from representation theory of finite dimensional algebras. Indeed, we may expect that various properties of the usual Hecke algebras also hold for the cyclotomic quiver Hecke algebras, and we may use the new proof techniques to prove them. The property we consider in our series of papers is the representation type. For the classical Hecke algebras associated with the symmetric group, there is a theorem by Erdmann and Nakano, so we study its analogue in other affine Lie types, namely Erdmann-Nakano type theorems for finite quiver Hecke algebras $R^{\Lambda_{0}}(\beta)$. In the first paper 2 of our series, we gave a general framework to determine the representation type, which

Received by the editors June 17, 2013 and, in revised form, February 24, 2014.

2010 Mathematics Subject Classification. Primary 16G60, 17B67, 81R10.

The first author was supported in part by JSPS, Grant-in-Aid for Scientific Research (B) 23340006 . 
gave a new proof to the original Erdmann-Nakano theorem, and we showed that the representation type in affine type $A_{2 \ell}^{(2)}$ was also described by Lie theoretic terms.

In this paper, we study affine Lie type $D_{\ell+1}^{(2)}$. We follow the general strategy established in [2]. Recall that a key result in [2] was the explicit description of irreducible $R^{\Lambda_{0}}\left(\delta-\alpha_{i}\right)$-modules. They were used to show that $R^{\Lambda_{0}}(\delta)$ is a Brauer tree algebra. The modules were homogeneous modules. In type $D_{\ell+1}^{(2)}$, a key achievement is our success in giving an explicit description of irreducible $R^{\Lambda_{0}}\left(2 \delta-\alpha_{i}\right)$-modules. The modules are no longer homogeneous, and their construction required more insight than $A_{\ell}^{(1)}$ and $A_{2 \ell}^{(2)}$. Then we use the explicit description to show that $R^{\Lambda_{0}}(2 \delta)$ is a symmetric special biserial algebra. We may determine the shape of the stable Auslander-Reiten quiver of finite quiver Hecke algebras of tame representation type. Other parts of the arguments in [2] may be carried out for type $D_{\ell+1}^{(2)}$ with slight modifications.

The paper is organized as follows. $\S 1$ and $\S 2$ are for preliminaries. In $\S 3$ we prove explicit dimension formulas for various spherical subalgebras of $R^{\Lambda_{0}}(n)$. The formulas are crucial for proving the main theorem, and they are repeatedly used in the remaining half of the paper. In $\S 4$ we construct various modules, and then we prove our main theorem in $\S 5$.

\section{Preliminaries}

In this section, we briefly recall the combinatorial realization of the highest weight $U_{q}\left(D_{\ell+1}^{(2)}\right)$-crystal $B\left(\Lambda_{0}\right)$ using Young walls and the Fock space of neutral fermions in aspect of $U\left(D_{\ell+1}^{(2)}\right)$-modules. We will use them to describe the categorification theory for cyclotomic quiver Hecke algebras of type $D_{\ell+1}^{(2)}$.

1.1. Cartan datum. Let $I=\{0,1, \ldots, \ell\}$ be an index set, and let A be the affine Cartan matrix of type $D_{\ell+1}^{(2)}(\ell \geq 2)$ :

$$
\mathrm{A}=\left(a_{i j}\right)_{i, j \in I}=\left(\begin{array}{ccccccc}
2 & -2 & 0 & \ldots & 0 & 0 & 0 \\
-1 & 2 & -1 & \ldots & 0 & 0 & 0 \\
0 & -1 & 2 & \ldots & 0 & 0 & 0 \\
\vdots & \vdots & \vdots & \ddots & \vdots & \vdots & \vdots \\
0 & 0 & 0 & \ldots & 2 & -1 & 0 \\
0 & 0 & 0 & \ldots & -1 & 2 & -1 \\
0 & 0 & 0 & \ldots & 0 & -2 & 2
\end{array}\right) .
$$

When $\ell=1$, the affine Cartan matrix of type $D_{2}^{(2)}$ is defined to be that of type $A_{1}^{(1)}$, namely

$$
\mathrm{A}=\left(a_{i j}\right)_{i, j \in I}=\left(\begin{array}{cc}
2 & -2 \\
-2 & 2
\end{array}\right)
$$

An affine Cartan datum $\left(\mathrm{A}, \mathrm{P}, \Pi, \Pi^{\vee}\right)$ is given as

(1) $A$ is the affine Cartan matrix as above,

(2) $\mathrm{P}$ is a free abelian group of rank $\ell+2$, called the weight lattice,

(3) $\Pi=\left\{\alpha_{i} \mid i \in I\right\} \subset \mathrm{P}$, called the set of simple roots,

(4) $\Pi^{\vee}=\left\{h_{i} \mid i \in I\right\} \subset \mathrm{P}^{\vee}:=\operatorname{Hom}(\mathrm{P}, \mathbb{Z})$, called the set of simple coroots, 
which satisfy the following properties:

(a) $\left\langle h_{i}, \alpha_{j}\right\rangle=a_{i j}$ for all $i, j \in I$,

(b) $\Pi$ and $\Pi^{\vee}$ are linearly independent sets.

As in [11, p. 21], we fix a scaling element $d$ which obeys the condition $\left\langle d, \alpha_{i}\right\rangle=\delta_{i 0}$, and assume that $\Pi^{\vee}$ and $d$ form a $\mathbb{Z}$-basis of $P^{\vee}$. Then, the fundamental weight $\Lambda_{0}$ is defined by

$$
\left\langle h_{i}, \Lambda_{0}\right\rangle=\delta_{i 0}, \quad\left\langle d, \Lambda_{0}\right\rangle=0 .
$$

We define another element $d \in \mathrm{P}^{\vee} \otimes_{\mathbb{Z}} \mathbb{Q}$ by

$$
\mathrm{d}=\sum_{i=1}^{\ell-1} i h_{i}+\frac{\ell}{2} h_{\ell}+2 d
$$

Then, this satisfies

$$
\left\langle\mathrm{d}, \Lambda_{0}\right\rangle=0, \quad\left\langle\mathrm{~d}, \alpha_{i}\right\rangle= \begin{cases}1 & \text { if } i=0, \ell \\ 0 & \text { otherwise }\end{cases}
$$

The free abelian group $\mathrm{Q}=\bigoplus_{i \in I} \mathbb{Z} \alpha_{i}$ is called the root lattice, and $\mathrm{Q}^{+}=$ $\sum_{i \in I} \mathbb{Z}_{\geq 0} \alpha_{i}$ is the positive cone of the root lattice. For $\beta=\sum_{i \in I} k_{i} \alpha_{i} \in \mathrm{Q}^{+}$, set $|\beta|=\sum_{i \in I} k_{i}$ to be the height of $\beta$. Let $\mathrm{W}$ be the Weyl group associated with A, which is generated by $\left\{r_{i}\right\}_{i \in I}$ acting on $P$ by $r_{i} \Lambda=\Lambda-\left\langle h_{i}, \Lambda\right\rangle \alpha_{i}$, for $\Lambda \in P$. The null root of type $D_{\ell+1}^{(2)}$ is

$$
\delta=\alpha_{0}+\alpha_{1}+\cdots+\alpha_{\ell-1}+\alpha_{\ell}
$$

Note that $\left\langle h_{i}, \delta\right\rangle=0$ and $w \delta=\delta$, for $i \in I$ and $w \in \mathrm{W}$. The standard symmetric bilinear pairing $(\mid)$ on $\mathrm{P}$ in type $D_{\ell+1}^{(2)}$ is

$$
\left(\alpha_{i} \mid \Lambda\right)=d_{i}\left\langle h_{i}, \Lambda\right\rangle \text { for all } \Lambda \in \mathrm{P},
$$

where $\left(d_{0}, d_{1}, \ldots, d_{\ell}\right)=(1,2, \ldots, 2,1)$.

1.2. Young walls. In this subsection, we review combinatorics of Young walls for type $D_{\ell+1}^{(2)}$. A Young wall is a generalization of a colored Young diagram, which gives a combinatorial realization of crystals for basic representations of various quantum affine algebras [11,14, 16, 17.

A Young wall of type $D_{\ell+1}^{(2)}$ is a wall consisting of the colored blocks below:

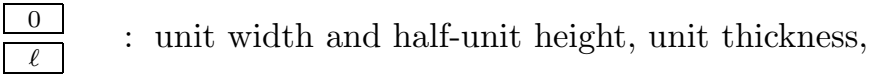

${ }_{i}(i=1, \ldots, \ell-1):$ unit width and unit height, unit thickness, 
built by the following rules:

(1) Blocks should be built in the pattern given below.

(2) There should be no free space to the right of any block except the rightmost column.

The pattern is given as follows:

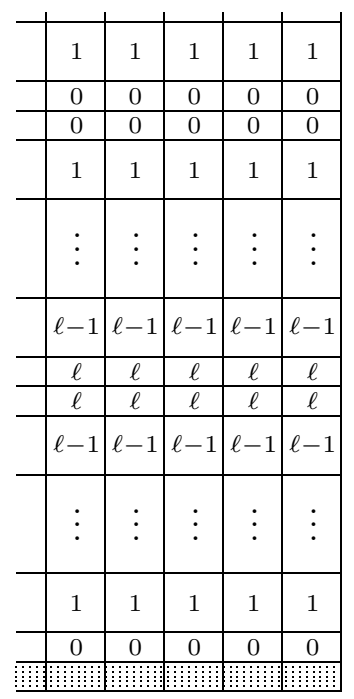

Note that the sequence $(0,1,2, \ldots, \ell-1, \ell, \ell, \ell-1, \ldots, 2,1,0)$ is repeated in each column.

A full column is a column whose height is a multiple of the unit length, and a $\delta$-column is a column consisting of two 0 -blocks, two 1 -blocks, ..., and two $\ell$-blocks. The definition of the $\delta$-column might confuse the reader, but recall that a $\delta$-column in type $D_{\ell+1}^{(2)}$ is a column of weight $2 \delta$. See [11, p. 279]. A Young wall is called proper if none of the full columns have the same height. A column in a proper Young wall is said to have a removable $\delta$ if we may remove a $\delta$-column from the given Young wall and still have a proper Young wall.

Let $\mathcal{Y}\left(\Lambda_{0}\right)$ be the set of all proper Young walls whose columns have no removable $\delta$. Kashiwara operators $\tilde{e}_{i}$ and $\tilde{f}_{i}$ on $\mathcal{Y}\left(\Lambda_{0}\right)$ can be defined by considering combinatorics of Young walls, which give a crystal structure of a quantum affine algebra $U_{q}(\mathfrak{g})$ of type $D_{\ell+1}^{(2)}[11,14$.

Theorem 1.1 ([14, Thm. 7.1]). The crystal $\mathcal{Y}\left(\Lambda_{0}\right)$ is isomorphic to the crystal $B\left(\Lambda_{0}\right)$ of the highest weight $U_{q}(\mathfrak{g})$-module $V_{q}\left(\Lambda_{0}\right)$.

Lemma 1.2. $\Lambda_{0}-2 \delta+\alpha_{i}$, for $0 \leq i \leq \ell-1$, are extremal weights.

Proof. Explicit computation shows

$$
\begin{aligned}
r_{1} r_{2} \cdots r_{\ell} \cdots r_{1} r_{0} \Lambda_{0} & =\Lambda_{0}-2 \delta+\alpha_{0}, \\
r_{2} \cdots r_{\ell} \cdots r_{2} r_{0} r_{1} r_{0} \Lambda_{0} & =\Lambda_{0}-2 \delta+\alpha_{1} .
\end{aligned}
$$

Then $r_{i} r_{i+1}\left(\Lambda_{0}-2 \delta+\alpha_{i}\right)=\Lambda_{0}-2 \delta+\alpha_{i+1}$, for $1 \leq i \leq \ell-2$, proves the result. 
Using the Young wall realization, one can observe the following facts:

(i) For each $i \in I, \mathcal{Y}\left(\Lambda_{0}\right)_{\Lambda_{0}-2 \delta+\alpha_{i}}$ consists of the element $Y_{i}$ given below.
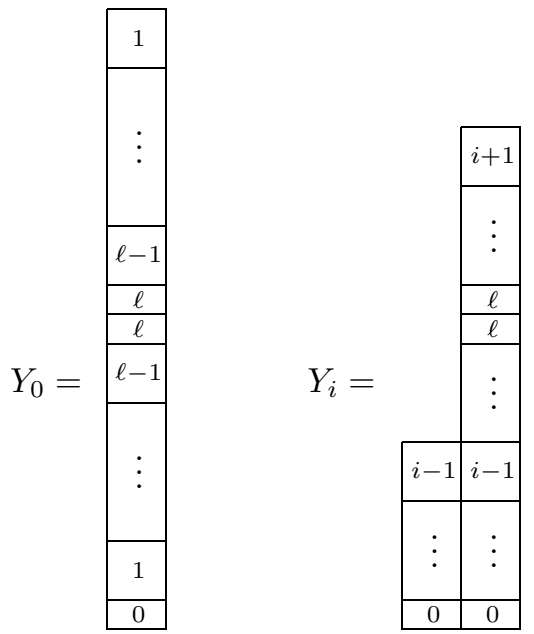

$$
(i=1, \ldots, \ell-1)
$$

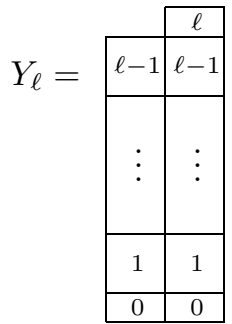

(ii) The Young walls $Y_{0}, \ldots, Y_{\ell-1}$ are extremal.

(iii) We have $\varepsilon_{\ell}\left(Y_{\ell-1}\right)=2$ and if $i, j \in I$ are such that $(i, j) \neq(\ell-1, \ell)$, then

$$
\varepsilon_{j}\left(Y_{i}\right)= \begin{cases}1 & \text { if } j=i-1, i+1 \\ 0 & \text { otherwise. }\end{cases}
$$

Moreover, in the case that $j$ is either $i-1$ or $i+1$, we have $\tilde{e}_{i}\left(Y_{j}\right)=\tilde{e}_{j}\left(Y_{i}\right)$.

(iv) We have

$$
\left|\mathcal{Y}\left(\Lambda_{0}\right)_{\Lambda_{0}-2 \delta}\right|=\ell+1
$$

1.3. Shifted Young diagrams. Let $\lambda=\left(\lambda_{1}>\lambda_{2}>\ldots>\lambda_{l}>0\right)$ be a shifted Young diagram of depth $l$. We denote the depth $l$ by $l(\lambda)$ and write $\lambda \vdash n$ if $\lambda$ consists of $n$ boxes. A tableau $T$ of shape $\lambda \vdash n$ is a filling of boxes of $\lambda$ with numbers $1, \ldots, n$, one for each box. For a simple transposition $s_{k}$, let $s_{k} T$ be the tableau obtained from $T$ by exchanging the entries $k$ and $k+1$. A standard tableau is a tableau whose entries in rows and columns increase from left to right and top to bottom, respectively. The canonical tableau $T^{\lambda}$ is the standard tableau whose $(i, j)$-entry is $(j-i)+1+\sum_{k=1}^{i-1} \lambda_{k}$. We denote by $\mathrm{ST}(\lambda)$ the set of all standard tableaux of shape $\lambda$. For example, the following are standard tableaux of shape $(5,2)$ :

\begin{tabular}{|l|l|l|l|l|}
\hline 1 & 2 & 4 & 6 & 7 \\
\hline & 3 & 5 & \multicolumn{2}{|c}{} \\
\cline { 2 - 5 } & &
\end{tabular}

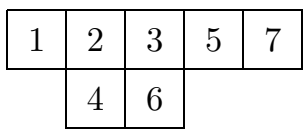

Let $\lambda$ be a shifted Young diagram. We consider the residue pattern $(\ell \geq 1)$

$$
012 \ldots \ell-1 \ell \ell \ell-1 \ldots 210,
$$

which repeats from left to right in each row of $\lambda$, and denote by $\operatorname{res}(i, j)$ the residue of the $(i, j)$-box in $\lambda$. For example, if $\ell=3$ and $\lambda=(12,7,3,2)$, the residues are 
given as follows:

\begin{tabular}{|c|c|c|c|c|c|c|c|c|c|c|c|}
\hline 0 & 1 & 2 & 3 & 3 & 2 & 1 & 0 & 0 & 1 & 2 & 3 \\
\hline & \multirow[t]{3}{*}{0} & 1 & 2 & 3 & 3 & 2 & 1 & & & & \\
\hline & & 0 & 1 & 2 & & & & & & & \\
\hline & & & 0 & 1 & & & & & & & \\
\hline
\end{tabular}

In this example, we have $\operatorname{res}(2,7)=2$. Note that the residue pattern also appears in columns of Young walls as colors (see Section 1.2).

Definition 1.3. For $T \in \mathrm{ST}(\lambda)$, we define the residue sequence of $T$ by

$$
\operatorname{res}(T)=\left(\operatorname{res}_{1}(T), \operatorname{res}_{2}(T), \ldots, \operatorname{res}_{n}(T)\right) \in I^{n},
$$

where $\operatorname{res}_{k}(T)$ is the residue of the box filled with $k$ in $T$, for $1 \leq k \leq n$.

1.4. The Fock space of neutral fermions. Let $C$ be the Clifford algebra over $\mathbb{C}$ generated by $\phi_{k}(k \in \mathbb{Z})$ with the following defining relations:

$$
\phi_{p} \phi_{q}+\phi_{q} \phi_{p}= \begin{cases}2 & \text { if } p=q=0 \\ (-1)^{p} \delta_{p,-q} & \text { otherwise }\end{cases}
$$

Consider the quotient space $\mathrm{F}=\mathrm{C} / \mathrm{I}$ by the left ideal $\mathrm{I}$ of $\mathrm{C}$ generated by $\left\{\phi_{k} \mid\right.$ $k<0\}$. For a shifted Young diagram $\lambda$, we can write it as $\lambda=\left(\lambda_{1}>\lambda_{2}>\ldots>\right.$ $\lambda_{2 r-1}>\lambda_{2 r} \geq 0$ ) for a unique number $r$, where $\lambda_{2 r}>0$ if $l(\lambda)$ is even and $\lambda_{2 r}=0$ if $l(\lambda)$ is odd. Set $|0\rangle=1+\mathrm{I}$ and

$$
|\lambda\rangle=\phi_{\lambda_{1}} \phi_{\lambda_{2}} \cdots \phi_{\lambda_{2 r}}|0\rangle \text {. }
$$

Note that $\{|\lambda\rangle \mid \lambda$ : shifted Young diagrams $\}$ is a linearly independent set in F.

We now define

$$
\mathcal{F}=\operatorname{Span}_{\mathbb{C}}\{|\lambda\rangle \mid \lambda: \text { shifted Young diagrams }\} \subseteq F,
$$

and describe the actions of the Chevalley generators $f_{i}, e_{i}(i \in I)$ on $\mathcal{F}$ for the Kac-Moody algebra $\mathfrak{g}$ associated with $\mathrm{A}$ by the reduction $(B K P)_{2 \ell+2}$ in [7,12] as follows:

$$
f_{i}=\sum_{j \geq 0, j \equiv i,-i-1} \mathrm{f}_{j}, \quad e_{i}= \begin{cases}\mathrm{e}_{0}+2 \sum_{j>0, j \equiv 0,-1} \mathrm{e}_{j} & \text { if } i=0, \\ \sum_{j>0, j \equiv i,-i-1} \mathrm{e}_{j} & \text { if } i=1, \ldots, \ell-1, \\ 2 \sum_{j>0, j \equiv \ell, \ell+1} \mathrm{e}_{j} & \text { if } i=\ell\end{cases}
$$

where all congruences are taken modulo $h:=2 \ell+2$, and

$$
\begin{aligned}
& \mathrm{e}_{0}=\phi_{-1} \phi_{0}, \quad \mathrm{e}_{j}=(-1)^{j} \phi_{-j-1} \phi_{j} \quad(j \geq 1), \\
& \mathrm{f}_{0}=\phi_{1} \phi_{0}, \quad \mathrm{f}_{j}=(-1)^{j} \phi_{j+1} \phi_{-j} \quad(j \geq 1) .
\end{aligned}
$$

$\mathcal{F}$ has the highest weight $\Lambda_{0}$. It is straightforward to check that if $\lambda$ has a row of length $j+1$ and does not have a row of length $j$ (resp. $\lambda$ has a row of length $j$ and does not have a row of length $j+1$ ), then we have

$$
\mathrm{e}_{j}|\lambda\rangle=\left|\mathrm{e}_{j} \lambda\right\rangle \quad\left(\text { resp. } \mathrm{f}_{j}|\lambda\rangle=\left|\mathrm{f}_{j} \lambda\right\rangle\right)
$$

where $e_{j} \lambda$ (resp. $f_{j} \lambda$ ) is the shifted Young diagram obtained from $\lambda$ by removing the rightmost box of the row of length $j+1$ (resp. adding a new box on the right of 
the row of length $j$ ). Otherwise, we have $\mathrm{e}_{j}|\lambda\rangle=0$ (resp. $\mathrm{f}_{j}|\lambda\rangle=0$ ). For a shifted Young diagram $\lambda,|\lambda\rangle$ is a weight vector whose weight is

$$
\operatorname{wt}(\lambda):=\operatorname{wt}(|\lambda\rangle)=\Lambda_{0}-\sum_{k \in \operatorname{res}(\lambda)} \alpha_{k}
$$

where $\operatorname{res}(\lambda)$ is a multiset whose elements are $\operatorname{res}(i, j)$ for all $(i, j) \in \lambda$.

\section{Quiver Hecke algebras}

In this section, we introduce the quiver Hecke algebras, the main object of study. Then we review the categorification of integrable highest weight modules and crystals using their module categories. The results in this section will be used for proving our main theorems. Throughout the paper, $\mathbf{k}$ is an algebraically closed field, and algebras are unital associative $\mathbf{k}$-algebras.

Let $\left(\mathrm{A}, \mathrm{P}, \Pi, \Pi^{\vee}\right)$ be the affine Cartan datum from Section 1.1, and take polynomials $\mathcal{Q}_{i, j}(u, v) \in \mathbf{k}[u, v]$, for $i, j \in I$, of the form

$$
\mathcal{Q}_{i, j}(u, v)= \begin{cases}\sum_{p\left(\alpha_{i} \mid \alpha_{i}\right)+q\left(\alpha_{j} \mid \alpha_{j}\right)+2\left(\alpha_{i} \mid \alpha_{j}\right)=0} t_{i, j ; p, q} u^{p} v^{q} & \text { if } i \neq j, \\ 0 & \text { if } i=j,\end{cases}
$$

where $t_{i, j ; p, q} \in \mathbf{k}$ are such that $t_{i, j ;-a_{i j}, 0} \neq 0$ and $\mathcal{Q}_{i, j}(u, v)=\mathcal{Q}_{j, i}(v, u)$. The symmetric group $\mathfrak{S}_{n}=\left\langle s_{k} \mid k=1, \ldots, n-1\right\rangle$ acts on $I^{n}$ by place permutations.

Definition 2.1. Let $\Lambda \in \mathrm{P}^{+}$. The cyclotomic quiver Hecke algebra $R^{\Lambda}(n)$ associated with polynomials $\left(\mathcal{Q}_{i, j}(u, v)\right)_{i, j \in I}$ and the dominant integral weight $\Lambda$ is the $\mathbb{Z}$-graded k-algebra defined by three sets of generators:

$$
\left\{e(\nu) \mid \nu=\left(\nu_{1}, \ldots, \nu_{n}\right) \in I^{n}\right\},\left\{x_{k} \mid 1 \leq k \leq n\right\},\left\{\psi_{l} \mid 1 \leq l \leq n-1\right\}
$$

subject to the following relations:

$$
\begin{aligned}
& e(\nu) e\left(\nu^{\prime}\right)=\delta_{\nu, \nu^{\prime}} e(\nu), \sum_{\nu \in I^{n}} e(\nu)=1, x_{k} e(\nu)=e(\nu) x_{k}, x_{k} x_{l}=x_{l} x_{k}, \\
& \psi_{l} e(\nu)=e\left(s_{l}(\nu)\right) \psi_{l}, \psi_{k} \psi_{l}=\psi_{l} \psi_{k} \text { if }|k-l|>1, \\
& \psi_{k}^{2} e(\nu)=\mathcal{Q}_{\nu_{k}, \nu_{k+1}}\left(x_{k}, x_{k+1}\right) e(\nu), \\
& \left(\psi_{k} x_{l}-x_{s_{k}(l)} \psi_{k}\right) e(\nu)= \begin{cases}-e(\nu) & \text { if } l=k \text { and } \nu_{k}=\nu_{k+1}, \\
e(\nu) & \text { if } l=k+1 \text { and } \nu_{k}=\nu_{k+1}, \\
0 & \text { otherwise },\end{cases} \\
& \left(\psi_{k+1} \psi_{k} \psi_{k+1}-\psi_{k} \psi_{k+1} \psi_{k}\right) e(\nu) \\
& = \begin{cases}\frac{\mathcal{Q}_{\nu_{k}, \nu_{k+1}}\left(x_{k}, x_{k+1}\right)-\mathcal{Q}_{\nu_{k}, \nu_{k+1}}\left(x_{k+2}, x_{k+1}\right)}{x_{k}-x_{k+2}} e(\nu) & \text { if } \nu_{k}=\nu_{k+2}, \\
0 & \text { otherwise, }\end{cases} \\
& x_{1}^{\left\langle h_{\nu_{1}}, \Lambda\right\rangle} e(\nu)=0 .
\end{aligned}
$$


The $\mathbb{Z}$-grading on $R^{\Lambda}(n)$ is given as follows:

$$
\operatorname{deg}(e(\nu))=0, \quad \operatorname{deg}\left(x_{k} e(\nu)\right)=\left(\alpha_{\nu_{k}} \mid \alpha_{\nu_{k}}\right), \quad \operatorname{deg}\left(\psi_{l} e(\nu)\right)=-\left(\alpha_{\nu_{l}} \mid \alpha_{\nu_{l+1}}\right) .
$$

For $\beta \in \mathrm{Q}^{+}$with $|\beta|=n$, we set $I^{\beta}=\left\{\nu=\left(\nu_{1}, \ldots, \nu_{n}\right) \in I^{n} \mid \sum_{k=1}^{n} \alpha_{\nu_{k}}=\beta\right\}$ and

$$
e(\beta)=\sum_{\nu \in I^{\beta}} e(\nu)
$$

which is a central idempotent of $R^{\Lambda}(n)$ since $I^{\beta}$ is invariant under the action of $\mathfrak{S}_{n}$. Define

$$
R^{\Lambda}(\beta)=R^{\Lambda}(n) e(\beta) .
$$

We do not know whether $R^{\Lambda}(\beta)$ is indecomposable. For an $R^{\Lambda}(\beta)$-module $M$, the $q$-character $\operatorname{ch}_{q}(M)$ is defined by

$$
\operatorname{ch}_{q}(M):=\sum_{\nu \in I^{\beta}} \operatorname{dim}_{q}(e(\nu) M) \nu,
$$

where $\operatorname{dim}_{q}(N):=\sum_{i \in \mathbb{Z}}\left(\operatorname{dim} N_{i}\right) q^{i}$ for a graded vector space $N=\bigoplus_{i \in \mathbb{Z}} N_{i}$.

We will be interested in the special cyclotomic quiver Hecke algebra $R^{\Lambda_{0}}(\beta)$ associated with the Cartan datum of type $D_{\ell+1}^{(2)}$, which we call finite quiver Hecke algebras of type $D_{\ell+1}^{(2)}$.

Remark 2.2. As is explained in the introduction, we focus on finite quiver Hecke algebras because they resemble the Hecke algebras associated with the symmetric group, and we study how far the properties which were found for the classical Hecke algebras remain true for finite quiver Hecke algebras. For example, we have already shown that the representation type follows Erdmann and Nakano's pattern in types $A_{\ell}^{(1)}, A_{2 \ell}^{(2)}$ and $D_{\ell+1}^{(2)}$, but in the fourth paper in our series, we will show that we may still use our method but the representation type follows a different pattern in type $C_{\ell}^{(1)}$. The Erdmann and Nakano pattern also fails for the level two and higher level cyclotomic Hecke algebras.

Before studying the finite quiver Hecke algebras of type $D_{\ell+1}^{(2)}$, we recall results which are valid for general $R^{\Lambda}(\beta)$.

Proposition 2.3 (cf. [2, Lem. 2.2]). The algebra $R^{\Lambda}(\beta)$ is finite dimensional and $x_{1}, \ldots, x_{n}$ are nilpotent.

Proposition 2.4 (cf. [2, Prop. 4.2]). The algebra $R^{\Lambda}(\beta)$ is a self-injective algebra.

Proposition 2.5 ([2, Cor. 4.8]). For $w \in \mathrm{W}$ and $k \in \mathbb{Z}_{\geq 0}, R^{\Lambda}(k \delta)$ and $R^{\Lambda}(\Lambda-w \Lambda+k \delta)$ have the same number of simple modules and the same representation type.

Let $\mathbb{A}=\mathbb{Z}\left[q, q^{-1}\right]$. We denote by $U_{\mathbb{A}}(\mathfrak{g})$ the $\mathbb{A}$-form of the quantum group $U_{q}(\mathfrak{g})$ associated with the Cartan datum $\left(\mathrm{A}, \mathrm{P}, \Pi, \Pi^{\vee}\right)$ and by $V_{\mathbb{A}}(\Lambda)$ the $\mathbb{A}$-form of highest weight $U_{q}(\mathfrak{g})$-module $V_{q}(\Lambda)$, respectively.

Let us denote the direct sum of split Grothendieck groups of additive categories $R^{\Lambda}(\beta)$-proj ${ }^{\mathbb{Z}}$ of finitely generated projective graded left $R^{\Lambda}(\beta)$-modules by

$$
K_{0}^{\mathbb{Z}}\left(R^{\Lambda}\right)=\bigoplus_{\beta \in Q^{+}} K_{0}\left(R^{\Lambda}(\beta)-\operatorname{proj}^{\mathbb{Z}}\right)
$$

Then, $K_{0}^{\mathbb{Z}}\left(R^{\Lambda}\right)$ has an $\mathbb{A}$-module structure induced by the $\mathbb{Z}$-grading on $R^{\Lambda}(n)$. 
Let $e\left(\nu, \nu^{\prime}\right)$ be the idempotent corresponding to the concatenation of $\nu$ and $\nu^{\prime}$. When $\nu^{\prime}=i$, we simply write $e(\nu, i)=e(\nu,(i))$ and $e(\beta, i)=\sum_{\nu \in I^{\beta}} e(\nu, i)$. For $\beta \in \mathrm{Q}^{+}$and $i \in I$, we define functors between categories of finitely generated graded modules as follows:

$$
\begin{array}{ll}
E_{i}: R^{\Lambda}\left(\beta+\alpha_{i}\right)-\bmod ^{\mathbb{Z}} \longrightarrow R^{\Lambda}(\beta)-\bmod ^{\mathbb{Z}}, & N \mapsto e(\beta, i) N, \\
F_{i}: R^{\Lambda}(\beta)-\bmod ^{\mathbb{Z}} \longrightarrow R^{\Lambda}\left(\beta+\alpha_{i}\right)-\bmod ^{\mathbb{Z}}, & M \mapsto R^{\Lambda}\left(\beta+\alpha_{i}\right) e(\beta, i) \otimes_{R^{\Lambda}(\beta)} M .
\end{array}
$$

Theorem 2.6 ([15, Thm. 5.2]). Let $l_{i}=\left\langle h_{i}, \Lambda-\beta\right\rangle$, for $i \in I$. Then one of the following isomorphisms of endofunctors holds.

(1) If $l_{i} \geq 0$, then

$$
q_{i}^{-2} F_{i} E_{i} \oplus \bigoplus_{k=0}^{l_{i}-1} q_{i}^{2 k} \text { id } \stackrel{\sim}{\longrightarrow} E_{i} F_{i} .
$$

(2) If $l_{i} \leq 0$, then

$$
q_{i}^{-2} F_{i} E_{i} \stackrel{\sim}{\longrightarrow} E_{i} F_{i} \oplus \bigoplus_{k=0}^{-l_{i}-1} q_{i}^{-2 k-2} \mathrm{id} .
$$

Moreover, $E_{i}$ and $F_{i}$ give a $U_{\mathbb{A}}(\mathfrak{g})$-module structure to $K_{0}^{\mathbb{Z}}\left(R^{\Lambda}\right)$.

Theorem 2.7 ([15, Thm. 6.2]). There exists a $U_{\mathbb{A}}(\mathfrak{g})$-module isomorphism between $K_{0}^{\mathbb{Z}}\left(R^{\Lambda}\right)$ and $V_{\mathbb{A}}(\Lambda)$.

Specializing $q \rightarrow 1$ on both sides of the isomorphism in Theorem 2.7, we have a $\mathfrak{g}$-module isomorphism

$$
V_{\mathbb{Z}}(\Lambda) \simeq K_{0}\left(R^{\Lambda}\right):=\bigoplus_{\beta \in Q^{+}} K_{0}\left(R^{\Lambda}(\beta) \text {-proj }\right),
$$

where $V_{\mathbb{Z}}(\Lambda)$ is the Kostant $\mathbb{Z}$-form of the highest weight module $V(\Lambda)$, and $R^{\Lambda}(\beta)$-proj is the category of finitely generated projective $R^{\Lambda}(\beta)$-modules.

By [18, Thm. 3.5] and [2, Lem. 3.3], the Shapovalov form (, ) of $V_{\mathbb{Z}}(\Lambda)$ can be understood as the dimension of the space of homomorphisms between projective modules. Namely, if $u, v \in V_{\mathbb{Z}}(\Lambda)_{\Lambda-\beta}$ correspond to actual projective $R^{\Lambda}(\beta)$-modules $U, V \in K_{0}\left(R^{\Lambda}(\beta)\right.$-proj) under the isomorphism (2.0.1), then we have

$$
(u, v)=\operatorname{dim} \operatorname{Hom}_{R^{\Lambda}(\beta)}(U, V) .
$$

Let $\mathbb{B}(\Lambda)$ be the set of all isomorphism classes of irreducible graded $R^{\Lambda}(\beta)$ modules, for all $\beta \in \mathrm{Q}^{+}$. For $i \in I$ and $L \in \mathbb{B}(\Lambda)$, the Kashiwara operators are defined by

$$
\tilde{e}_{i}(L)=\operatorname{Soc}\left(E_{i} L\right) \quad \text { and } \quad \tilde{f}_{i}(L)=\operatorname{Top}\left(F_{i} L\right),
$$

which give a $U_{q}(\mathfrak{g})$-crystal structure on $\mathbb{B}(\Lambda)$.

Theorem 2.8 ([22, Thm. 7.5]). The crystal $\mathbb{B}(\Lambda)$ is isomorphic to the crystal $B(\Lambda)$ of the highest weight $U_{q}(\mathfrak{g})$-module $V_{q}(\Lambda)$. 


\section{Dimension formula for $R^{\Lambda_{0}}(\beta)$}

In this section, we give a dimension formula for finite quiver Hecke algebras $R^{\Lambda_{0}}(\beta)$ in terms of combinatorics of shifted Young diagrams. The Fock space $\mathcal{F}$ in $\$ 1.4$ and the categorication (2.0.1) in $₫ 2$ are key ingredients for deducing the dimension formula.

For a shifted Young diagram $\lambda$,

- if we remove a box of residue $i$ from $\lambda$ and get a new shifted Young diagram, then we write $\lambda \nearrow i$ for the resulting diagram;

- if we add a box of residue $i$ to $\lambda$ and get a new shifted Young diagram, then we write $\lambda \swarrow i$ for the resulting diagram.

Then, it follows from (1.4.1) and (1.4.2) that

$$
e_{i}|\lambda\rangle=\sum_{\mu=\lambda \nearrow i} \mathrm{~m}(\lambda, \mu)|\mu\rangle, \quad f_{i}|\lambda\rangle=\sum_{\mu=\lambda \swarrow \swarrow i}|\mu\rangle,
$$

where

$$
\mathrm{m}(\lambda, \mu)= \begin{cases}2 & \text { if either }\left(\operatorname{wt}(\mu)=\operatorname{wt}(\lambda)+\alpha_{0} \text { and } l(\lambda)=l(\mu)\right) \\ 1 & \text { or }\left(\operatorname{wt}(\mu)=\operatorname{wt}(\lambda)+\alpha_{\ell}\right),\end{cases}
$$

The coefficients appearing in (3.0.1) are different from those in [2, (3.1.1)]. The reduction processes using the Fock spaces for type $A_{2 \ell}^{(2)}$ and type $D_{\ell+1}^{(2)}$ give that difference, which yields different dimension formulas for $R^{\Lambda_{0}}(\beta)$.

For $\lambda \vdash n$ and $\nu \in I^{n}$, we set

$$
K(\lambda, \nu)=|\{T \in \mathrm{ST}(\lambda) \mid \nu=\operatorname{res}(T)\}|
$$

so that

$$
|\mathrm{ST}(\lambda)|=\sum_{\nu \in I^{\Lambda_{0}-\mathrm{wt}(\lambda)}} K(\lambda, \nu) .
$$

Recall the element $\mathrm{d}$ defined in (1.1.1). Note that if $\operatorname{wt}(\lambda)=\Lambda_{0}-\sum_{i \in I} k_{i} \alpha_{i}$, then $k_{0}+k_{\ell}=-\langle\mathrm{d}, \operatorname{wt}(\lambda)\rangle$. Then, the following Lemma 3.1. Theorem 3.2 and Corollary 3.3 can be proved in the same manner as [2, Lem. 3.2, Thm. 3.4, Cor. 3.5].

Lemma 3.1. For $\lambda \vdash n$ and $\nu=\left(\nu_{1}, \nu_{2}, \ldots, \nu_{n}\right) \in I^{n}$, we obtain

$$
\begin{aligned}
e_{\nu_{1}} e_{\nu_{2}} \cdots e_{\nu_{n}}|\lambda\rangle & =2^{-\langle\mathrm{d}, \mathrm{wt}(\lambda)\rangle-l(\lambda)} K(\lambda, \nu)|0\rangle, \\
f_{\nu_{n}} f_{\nu_{n-1}} \cdots f_{\nu_{1}}|0\rangle & =\sum_{\mu \vdash n} K(\mu, \nu)|\mu\rangle .
\end{aligned}
$$

Theorem 3.2. Let $\lambda \vdash n$ be a shifted Young diagram. For $\beta \in \mathrm{Q}^{+}$with $|\beta|=n$ and $\nu, \nu^{\prime} \in I^{\beta}$, we have

$$
\begin{aligned}
\operatorname{dim} e\left(\nu^{\prime}\right) R^{\Lambda_{0}}(n) e(\nu) & =\sum_{\lambda \vdash n} 2^{-\langle\mathrm{d}, \mathrm{wt}(\lambda)\rangle-l(\lambda)} K\left(\lambda, \nu^{\prime}\right) K(\lambda, \nu), \\
\operatorname{dim} R^{\Lambda_{0}}(\beta) & =\sum_{\lambda \vdash n, \operatorname{wt}(\lambda)=\Lambda_{0}-\beta} 2^{-\langle\mathrm{d}, \mathrm{wt}(\lambda)\rangle-l(\lambda)}|\mathrm{ST}(\lambda)|^{2}, \\
\operatorname{dim} R^{\Lambda_{0}}(n) & =\sum_{\lambda \vdash n} 2^{-\langle\mathrm{d}, \operatorname{wt}(\lambda)\rangle-l(\lambda)}|\mathrm{ST}(\lambda)|^{2} .
\end{aligned}
$$


Corollary 3.3. (1) Let $\nu \in I^{n}$. Then, $e(\nu) \neq 0$ in $R^{\Lambda_{0}}(n)$ if and only if $\nu$ may be obtained from a standard tableau $T$ as $\nu=\operatorname{res}(T)$.

(2) We have the following hook length formula:

$$
\operatorname{dim} R^{\Lambda_{0}}(\beta)=\sum_{\lambda \vdash n, \operatorname{wt}(\lambda)=\Lambda_{0}-\beta} 2^{-\langle\mathrm{d}, \mathrm{wt}(\lambda)\rangle-l(\lambda)}\left(\frac{n !}{\prod_{(i, j) \in \lambda} h_{i, j}}\right)^{2},
$$

where $h_{i, j}$ is the hook length of $(i, j) \in \lambda$.

(3) For a natural number $n$, we have

$$
n !=\sum_{\beta \in \mathrm{Q}^{+},|\beta|=n} 2^{n-\langle\mathrm{d}, \beta\rangle} \operatorname{dim} R^{\Lambda_{0}}(\beta) .
$$

Remark 3.4. When $\ell=1$, Theorem 3.2 recovers the following classical combinatorial formula:

$$
n !=\sum_{\lambda \vdash n} 2^{n-l(\lambda)}|\mathrm{ST}(\lambda)|^{2} .
$$

The proof goes as follows. Suppose that $\ell=1$. Then, $-\langle\mathrm{d}$, wt $(\lambda)\rangle-l(\lambda)=n-l(\lambda)$ and Theorem 3.2 implies that

$$
\operatorname{dim} R^{\Lambda_{0}}(n)=\sum_{\lambda \vdash n} 2^{n-l(\lambda)}|\mathrm{ST}(\lambda)|^{2} .
$$

On the other hand, since $D_{\ell+1}^{(2)}$ for $\ell=1$ is $A_{1}^{(1)}$, there exists an isomorphism of algebras between $R^{\Lambda_{0}}(n)$ and the finite Hecke algebra $\mathcal{H}_{-1}\left(S_{n}\right)$ associated with the symmetric group $S_{n}$ and the parameter $q=-1$ [4,28]. Thus, $\operatorname{dim} R^{\Lambda_{0}}(n)=n$ ! and we are done.

Note that the proof of Corollary [3.3) (3) uses the formula, so that we must avoid its use in the above proof. For this reason, we use a different argument to relate $\operatorname{dim} R^{\Lambda_{0}}(n)$ and $n$ !.

\section{Representations of $R^{\Lambda_{0}}(\beta)$}

In this section, we give various representations for the algebras $R^{\Lambda_{0}}(\delta)$, $R^{\Lambda_{0}}\left(2 \delta-\alpha_{i}\right)$ and $R^{\Lambda_{0}}(2 \delta)$. Those representations will play crucial roles in proving our main theorem in $\$ 5$. We assume that $\ell \geq 2$. We also suppose that $\mathcal{Q}_{0,1}(u, v)=u^{2}+v, \mathcal{Q}_{\ell-1, \ell}(u, v)=u+v^{2}$ and $\mathcal{Q}_{i, j}(u, v)=1$ if $a_{i, j}=0$, for simplicity.

4.1. Representations of $R^{\Lambda_{0}}(\delta)$. We show that the algebra homomorphism

$$
\mathbf{k}[x] \rightarrow R^{\Lambda_{0}}(\delta): \quad x \mapsto x_{\ell+1}
$$

induces an isomorphism of algebras $\mathbf{k}[x] /\left(x^{2}\right) \simeq R^{\Lambda_{0}}(\delta)$.

Observe that the residue pattern (1.3.1) implies that $\lambda=(\ell+1)$ is the unique shifted Young diagram of weight $\Lambda_{0}-\delta$, and $\mathrm{ST}(\lambda)$ consists of the standard tableau

\begin{tabular}{|l|l|l|l|l|}
\hline 0 & 1 & $\cdots$ & $\ell-1$ & $\ell$ \\
\hline
\end{tabular}


Hence, Theorem 3.2 implies that $\operatorname{dim} R^{\Lambda_{0}}(\delta)=2$. Now, it is straightforward to check that

$$
\begin{aligned}
& e(\nu) \mapsto \begin{cases}\left(\begin{array}{ll}
1 & 0 \\
0 & 1
\end{array}\right) & \text { if } \nu=(0,1, \ldots, \ell), \\
\left(\begin{array}{ll}
0 & 0 \\
0 & 0
\end{array}\right) & \text { otherwise, }\end{cases} \\
& \psi_{j} \mapsto\left(\begin{array}{ll}
0 & 0 \\
0 & 0
\end{array}\right) \text {, } \\
& x_{i} \mapsto \begin{cases}\left(\begin{array}{ll}
0 & 1 \\
0 & 0
\end{array}\right) & \text { if } i=\ell+1, \\
\left(\begin{array}{ll}
0 & 0 \\
0 & 0
\end{array}\right) & \text { otherwise }\end{cases}
\end{aligned}
$$

is a well-defined representation of $R^{\Lambda_{0}}(\delta)$. Hence, we have the algebra isomorphism

$$
R^{\Lambda_{0}}(\delta) \simeq \mathbf{k}\left(\begin{array}{ll}
1 & 0 \\
0 & 1
\end{array}\right) \oplus \mathbf{k}\left(\begin{array}{ll}
0 & 1 \\
0 & 0
\end{array}\right) \simeq \mathbf{k}[x] /\left(x^{2}\right) .
$$

4.2. Representations of $R^{\Lambda_{0}}\left(2 \delta-\alpha_{i}\right)$. In this subsection, we study $R^{\Lambda_{0}}\left(2 \delta-\alpha_{i}\right)$. A key point here is that the irreducible $R^{\Lambda_{0}}\left(2 \delta-\alpha_{i}\right)$-module is unique and we may give its explicit description, for all $i \in I$. We will use the result to determine the structure of $R^{\Lambda_{0}}(2 \delta)$.

Let $h=2 \ell+2$ as before. We consider two rows partition $\lambda(i)=(h-i-1, i) \vdash h-1$ of weight $\Lambda_{0}-2 \delta+\alpha_{i}$, for $0 \leq i \leq \ell$. Then the residues of the nodes of $\lambda(i)$ are given as follows:

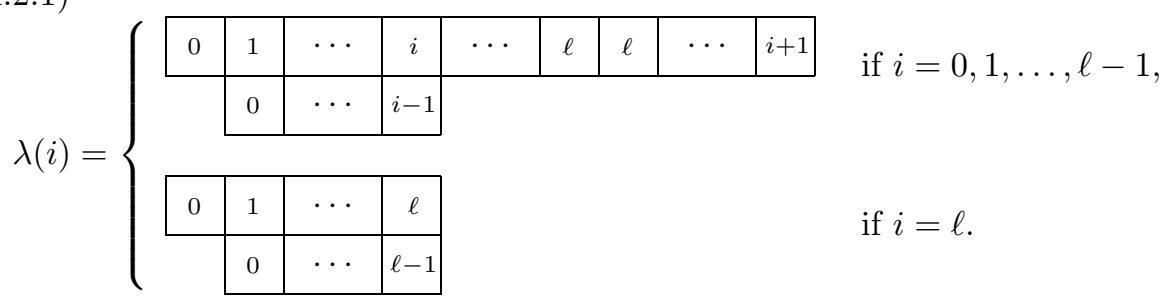

It is easy to check that $T \in \mathrm{ST}(\lambda(i))$ is uniquely determined by $\operatorname{res}(T)$. Let $\mathcal{N}=\mathbf{k}^{2}$ be the two dimensional vector space and define

$$
\xi_{1}=\left(\begin{array}{ll}
0 & 1 \\
0 & 0
\end{array}\right), \quad \xi_{2}=\left(\begin{array}{cc}
0 & -1 \\
0 & 0
\end{array}\right), \quad \partial=\left(\begin{array}{cc}
0 & 0 \\
-1 & 0
\end{array}\right)
$$

Remark 4.1. We have $\xi_{1} \partial-\partial \xi_{2}=-1=\partial \xi_{1}-\xi_{2} \partial, \xi_{1}^{2}=0, \xi_{1} \xi_{2}=\xi_{2} \xi_{1}$ and $\partial^{2}=0$. These are defining relations for the cyclotomic nilHecke algebra $\mathrm{NH}_{2}^{2}$. Indeed, $N H_{2}^{2}$ is isomorphic to $\operatorname{Mat}(2, \mathbf{k})$ [21, Prop. 5.3], and $\mathcal{N}$ is the unique irreducible $\mathrm{NH}_{2}^{2}$-module.

We shall define $R^{\Lambda_{0}}\left(2 \delta-\alpha_{i}\right)$-modules $\mathcal{L}_{i}$, for $0 \leq i \leq \ell$. Let

$$
\mathcal{L}_{i}=\bigoplus_{T \in \mathrm{ST}(\lambda(i))} \mathcal{N} \otimes T
$$

be the direct sum of copies of $\mathcal{N}$ such that each of the copies is labeled by $\operatorname{ST}(\lambda(i))$. 
Lemma 4.2. If $0 \leq i \leq \ell-1$, we may define an $R^{\Lambda_{0}}\left(2 \delta-\alpha_{i}\right)$-module structure on $\mathcal{L}_{i}$ by

$$
\begin{aligned}
e(\nu)(v \otimes T) & = \begin{cases}v \otimes T & \text { if } \nu=\operatorname{res}(T), \\
0 & \text { otherwise, }\end{cases} \\
x_{k}(v \otimes T) & = \begin{cases}\xi_{1} v \otimes T & \text { if } k \text { is located at }(1, \ell+1) \in \lambda(i), \\
\xi_{2} v \otimes T & \text { if } k \text { is located at }(1, \ell+2) \in \lambda(i), \\
0 & \text { otherwise, }\end{cases} \\
\psi_{k}(v \otimes T) & = \begin{cases}\partial v \otimes T & \text { if } k \text { and } k+1 \text { are located at }(1, \ell+1) \text { and }(1, \ell+2), \\
v \otimes s_{k} T & \text { if } s_{k} T \text { is standard, } \\
0 & \text { otherwise. }\end{cases}
\end{aligned}
$$

If $i=\ell$, we may define an $R^{\Lambda_{0}}\left(2 \delta-\alpha_{\ell}\right)$-module structure on $\mathcal{L}_{\ell}$ by

$$
\begin{aligned}
e(\nu)(v \otimes T) & = \begin{cases}v \otimes T & \text { if } \nu=\operatorname{res}(T), \\
0 & \text { otherwise, }\end{cases} \\
x_{k}(v \otimes T) & = \begin{cases}\xi_{1} v \otimes T & \text { if } k \text { is located at }(1, \ell+1) \in \lambda(\ell), \\
0 & \text { otherwise, }\end{cases} \\
\psi_{k}(v \otimes T) & = \begin{cases}v \otimes s_{k} T & \text { if } s_{k} T \text { is standard, } \\
0 & \text { otherwise. }\end{cases}
\end{aligned}
$$

Proof. To check the defining relations on $v \otimes T$, we may assume that

$$
\nu=\left(\nu_{1}, \nu_{2}, \ldots, \nu_{h-1}\right)=\operatorname{res}(T) .
$$

Then, using the residue pattern (1.3.1) and combinatorics of standard tableaux, we have the following:

(i) If $\nu_{k}=\nu_{k+1}$, then $0 \leq i \leq \ell-1$ and $\nu_{k}=\nu_{k+1}=\ell$.

(ii) $s_{k} T$ is standard if and only if $a_{\nu_{k}, \nu_{k+1}}=0$.

(iii) If $\nu_{k}=\nu_{k+2}$, then $\left(\nu_{k}, \nu_{k+1}, \nu_{k+2}\right)$ is either $(0,1,0)$ or $(\ell, j, \ell)$ for some $j \in I$ with $a_{\ell, j}=0$.

In the rest of the proof, we only check the relations for $\left(\psi_{k} x_{l}-x_{s_{k}(l)} \psi_{k}\right) e(\nu)$, $\psi_{k}^{2} e(\nu)$ and $\left(\psi_{k+1} \psi_{k} \psi_{k+1}-\psi_{k} \psi_{k+1} \psi_{k}\right) e(\nu)$, because it is straightforward to check other relations.

Let us start with $\left(\psi_{k} x_{l}-x_{s_{k}(l)} \psi_{k}\right) e(\nu)(v \otimes T)$. Suppose that $\nu_{k}=\nu_{k+1}$. Then (i) implies that $k$ and $k+1$ are located at the nodes $(1, \ell+1)$ and $(1, \ell+2)$ of $\lambda(i)$. If $l \neq k, k+1$, then

$$
x_{l}(v \otimes T)=0, \quad x_{s_{k}(l)} \psi_{k}(v \otimes T)=x_{l}(\partial v \otimes T)=0 .
$$

Thus, $\left(\psi_{k} x_{l}-x_{s_{k}(l)} \psi_{k}\right) e(\nu)(v \otimes T)=0$ as desired. If $l=k$, then

$$
\left(\psi_{k} x_{k}-x_{k+1} \psi_{k}\right) e(\nu)(v \otimes T)=\partial \xi_{1} v \otimes T-\xi_{2} \partial v \otimes T=-v \otimes T=-e(\nu)(v \otimes T) .
$$

If $l=k+1$, similar computation shows $\left(\psi_{k} x_{k+1}-x_{k} \psi_{k}\right) e(\nu)(v \otimes T)=e(\nu)(v \otimes T)$. Next we suppose that $\nu_{k} \neq \nu_{k+1}$ and prove $\left(\psi_{k} x_{l}-x_{s_{k}(l)} \psi_{k}\right) e(\nu)(v \otimes T)=0$. If $s_{k} T$ is not standard, then $\psi_{k}(\mathcal{N} \otimes T)=0$ implies the result. If $s_{k} T$ is standard, we define

$$
\eta_{l}(T)=\#\left\{1 \leq t \leq l \mid \operatorname{res}_{t}(T)=\operatorname{res}_{l}(T)\right\} .
$$

Then $\eta_{l}(T)$ is either 1 or 2 and we may write

$$
x_{l}(v \otimes T)=\delta_{\ell, \operatorname{res}_{l}(T)} \xi_{\eta_{l}(T)} v \otimes T .
$$


Since $\operatorname{res}_{l}(T)=\operatorname{res}_{s_{k}(l)}\left(s_{k} T\right)$ holds and $\nu_{k} \neq \nu_{k+1}$ implies $\eta_{l}(T)=\eta_{s_{k}(l)}\left(s_{k} T\right)$, we have

$$
\begin{aligned}
& \left(\psi_{k} x_{l}-x_{s_{k}(l)} \psi_{k}\right) e(\nu)(v \otimes T) \\
& \quad=\left(\delta_{\ell, \operatorname{res}_{l}(T)} \xi_{\eta_{l}(T)}-\delta_{\ell, \operatorname{res}_{s_{k}(l)}\left(s_{k} T\right)} \xi_{\eta_{s_{k}(l)}\left(s_{k} T\right)}\right)\left(v \otimes s_{k} T\right)=0 .
\end{aligned}
$$

Next we prove the relation for $\psi_{k}^{2} e(\nu)$. If $s_{k} T$ is standard, then $a_{\nu_{k}, \nu_{k+1}}=0$ by (ii), so that $\mathcal{Q}_{\nu_{k}, \nu_{k+1}}\left(x_{k}, x_{k+1}\right)=1$ and

$$
\psi_{k}^{2} e(\nu)(v \otimes T)=\mathcal{Q}_{\nu_{k}, \nu_{k+1}}\left(x_{k}, x_{k+1}\right) e(\nu)(v \otimes T)
$$

holds. If $s_{k} T$ is not standard, then $\psi_{k}^{2} e(\nu)(v \otimes T)=0$ and we have to verify $\mathcal{Q}_{\nu_{k}, \nu_{k+1}}\left(x_{k}, x_{k+1}\right) e(\nu)(v \otimes T)=0$. If $\nu_{k} \neq \ell$ and $\nu_{k+1} \neq \ell$, it is clear. Since $s_{k} T$ is not standard, the remaining cases are $\left(\nu_{k}, \nu_{k+1}\right)=(\ell, \ell)$ or $\left(\nu_{k}, \nu_{k+1}\right)=$ $(\ell, \ell-1),(\ell-1, \ell)$. If $\left(\nu_{k}, \nu_{k+1}\right)=(\ell, \ell)$, then $\mathcal{Q}_{\nu_{k}, \nu_{k+1}}\left(x_{k}, x_{k+1}\right)=0$ and there is nothing to prove. Suppose that $\left(\nu_{k}, \nu_{k+1}\right)=(\ell-1, \ell)$. Then

$$
\mathcal{Q}_{\ell-1, \ell}\left(x_{k}, x_{k+1}\right) e(\nu)(v \otimes T)=\left(x_{k}+x_{k+1}^{2}\right)(v \otimes T)=\xi_{\eta_{k+1}(T)}^{2} v \otimes T=0 .
$$

The proof for the case $\left(\nu_{k}, \nu_{k+1}\right)=(\ell, \ell-1)$ is similar.

Finally, we consider $\left(\psi_{k+1} \psi_{k} \psi_{k+1}-\psi_{k} \psi_{k+1} \psi_{k}\right) e(\nu)(v \otimes T)$. If

$$
\psi_{k+1} \psi_{k} \psi_{k+1}(v \otimes T) \neq 0,
$$

then one of the following holds and the other does not hold.

(a) $s_{k+1} T, s_{k} s_{k+1} T, s_{k+1} s_{k} s_{k+1} T$ are all standard.

(b) $\nu_{k}=\nu_{k+1}=\ell$ or $\nu_{k}=\nu_{k+2}=\ell$ or $\nu_{k+1}=\nu_{k+2}=\ell$.

Similarly, if $\psi_{k} \psi_{k+1} \psi_{k}(v \otimes T) \neq 0$, then one of the following holds and the other does not.

(a) $s_{k} T, s_{k+1} s_{k} T, s_{k} s_{k+1} s_{k} T$ are all standard.

(b) $\nu_{k}=\nu_{k+1}=\ell$ or $\nu_{k}=\nu_{k+2}=\ell$ or $\nu_{k+1}=\nu_{k+2}=\ell$.

Note that $s_{k} T, s_{k+1} s_{k} T, s_{k} s_{k+1} s_{k} T$ are all standard if and only if $s_{k+1} T, s_{k} s_{k+1} T$, $s_{k+1} s_{k} s_{k+1} T$ are all standard. Thus, there are two cases to consider. In case (a), we have

$$
\left(\psi_{k+1} \psi_{k} \psi_{k+1}-\psi_{k} \psi_{k+1} \psi_{k}\right)(v \otimes T)=0 .
$$

In case (b), $i \neq \ell$ and $\left(\nu_{k}, \nu_{k+1}, \nu_{k+2}\right)=(j, \ell, \ell),(\ell, j, \ell),(\ell, \ell, j)$, for some $j \in I$ with $j \neq \ell$. Using (ii), we have the following.

(1) If $\left(\nu_{k}, \nu_{k+1}, \nu_{k+2}\right)=(j, \ell, \ell)$ and $a_{j, \ell} \neq 0$, then

$$
\psi_{k}(v \otimes T)=0, \quad \psi_{k} \psi_{k+1}(v \otimes T)=\psi_{k}(\partial v \otimes T)=0 .
$$

(2) If $\left(\nu_{k}, \nu_{k+1}, \nu_{k+2}\right)=(j, \ell, \ell)$ and $a_{j, \ell}=0$, then

$$
\psi_{k+1} \psi_{k} \psi_{k+1}(v \otimes T)=\partial v \otimes s_{k+1} s_{k} T=\psi_{k} \psi_{k+1} \psi_{k}(v \otimes T) .
$$

(3) If $\left(\nu_{k}, \nu_{k+1}, \nu_{k+2}\right)=(\ell, j, \ell)$ and $a_{j, \ell} \neq 0$, then

$$
\psi_{k}(v \otimes T)=0, \quad \psi_{k+1}(v \otimes T)=0 .
$$

(4) If $\left(\nu_{k}, \nu_{k+1}, \nu_{k+2}\right)=(\ell, j, \ell)$ and $a_{j, \ell}=0$, then

$$
\psi_{k+1} \psi_{k} \psi_{k+1}(v \otimes T)=\partial v \otimes T=\psi_{k} \psi_{k+1} \psi_{k}(v \otimes T) .
$$

(5) If $\left(\nu_{k}, \nu_{k+1}, \nu_{k+2}\right)=(\ell, \ell, j)$ and $a_{j, \ell} \neq 0$, then

$$
\psi_{k+1}(v \otimes T)=0, \quad \psi_{k+1} \psi_{k}(v \otimes T)=\psi_{k+1}(\partial v \otimes T)=0 .
$$


(6) If $\left(\nu_{k}, \nu_{k+1}, \nu_{k+2}\right)=(\ell, \ell, j)$ and $a_{j, \ell}=0$, then

$$
\psi_{k+1} \psi_{k} \psi_{k+1}(v \otimes T)=\partial v \otimes s_{k} s_{k+1} T=\psi_{k} \psi_{k+1} \psi_{k}(v \otimes T) .
$$

Therefore, we always have $\left(\psi_{k+1} \psi_{k} \psi_{k+1}-\psi_{k} \psi_{k+1} \psi_{k}\right) e(\nu)(v \otimes T)=0$. Thus, it is enough to prove that if $\nu_{k}=\nu_{k+2}$, then

$$
\frac{\mathcal{Q}_{\nu_{k}, \nu_{k+1}}\left(x_{k}, x_{k+1}\right)-\mathcal{Q}_{\nu_{k}, \nu_{k+1}}\left(x_{k+2}, x_{k+1}\right)}{x_{k}-x_{k+2}}(v \otimes T)=0 .
$$

Suppose that $\nu_{k}=\nu_{k+2}$. Then (iii) implies that $\left(\nu_{k}, \nu_{k+1}, \nu_{k+2}\right)=(0,1,0)$ or $(\ell, j, \ell)$ with $a_{\ell, j}=0$. If $\left(\nu_{k}, \nu_{k+1}, \nu_{k+2}\right)=(0,1,0)$, then

$$
\frac{\mathcal{Q}_{0,1}\left(x_{k}, x_{k+1}\right)-\mathcal{Q}_{0,1}\left(x_{k+2}, x_{k+1}\right)}{x_{k}-x_{k+2}}(v \otimes T)=\left(x_{k}+x_{k+2}\right)(v \otimes T)=0 .
$$

If $\left(\nu_{k}, \nu_{k+1}, \nu_{k+2}\right)=(\ell, j, \ell)$ with $a_{\ell, j}=0$, then $\mathcal{Q}_{\ell, j}(u, v)=1$ and

$$
\frac{\mathcal{Q}_{\ell, j}\left(x_{k}, x_{k+1}\right)-\mathcal{Q}_{\ell, j}\left(x_{k+2}, x_{k+1}\right)}{x_{k}-x_{k+2}}(v \otimes T)=0 .
$$

We have completed the proof.

Corollary 4.3. Define

$$
\widetilde{\mathcal{L}}_{\ell}=\bigoplus_{T \in \mathrm{ST}(\lambda(\ell))} \mathbf{k}\left(\begin{array}{l}
1 \\
0
\end{array}\right) \otimes T
$$

Then $\widetilde{\mathcal{L}}_{\ell}$ is an $R^{\Lambda_{0}}\left(2 \delta-\alpha_{\ell}\right)$-submodule of $\mathcal{L}_{\ell}$ and we have a nonsplit exact sequence

$$
0 \longrightarrow \widetilde{\mathcal{L}}_{\ell} \longrightarrow \mathcal{L}_{\ell} \longrightarrow \widetilde{\mathcal{L}}_{\ell} \longrightarrow 0 .
$$

Proof. We prove that the exact sequence is nonsplit. The other parts are clear by (4.2.3). By definition, all $x_{k}$ act as 0 on $\widetilde{\mathcal{L}}_{\ell}$. Thus, $\mathcal{L}_{\ell} \neq \widetilde{\mathcal{L}}_{\ell} \oplus \widetilde{\mathcal{L}}_{\ell}$ because the action of $x_{k}$ is nonzero on $\mathcal{L}_{\ell}$.

Lemma 4.4. (1) The modules $\mathcal{L}_{i}$, for $0 \leq i \leq \ell-1$, and $\widetilde{\mathcal{L}}_{\ell}$ are irreducible.

(2) For $0 \leq i, j \leq \ell-1$, we have $E_{j} \mathcal{L}_{i}=0$ if and only if $j \neq i \pm 1$. Moreover,

$$
E_{i} \mathcal{L}_{j} \simeq E_{j} \mathcal{L}_{i},
$$

and they are irreducible if they are nonzero.

(3) $E_{i} \widetilde{\mathcal{L}}_{\ell}=0$ if and only if $i \neq \ell-1$, and $E_{\ell-1} \widetilde{\mathcal{L}}_{\ell}$ is irreducible.

(4) $E_{\ell-1} \mathcal{L}_{\ell}$ is isomorphic to $E_{\ell} \mathcal{L}_{\ell-1}$, and there is a nonsplit exact sequence

$$
0 \longrightarrow E_{\ell-1} \widetilde{\mathcal{L}}_{\ell} \longrightarrow E_{\ell} \mathcal{L}_{\ell-1} \longrightarrow E_{\ell-1} \widetilde{\mathcal{L}}_{\ell} \longrightarrow 0 .
$$

Proof. We show that $\mathcal{L}_{i}$, for $0 \leq i \leq \ell-1$, are irreducible. Let $W$ be a nonzero $R^{\Lambda_{0}}\left(2 \delta-\alpha_{i}\right)$-submodule of $\mathcal{L}_{i}$. Then

$$
W=\bigoplus_{T \in \mathbf{S T}(\lambda(i))} e(\operatorname{res}(T)) W
$$

and $e(\operatorname{res}(T)) W \neq 0$, for some $T \in \mathrm{ST}(\lambda(i))$. Noting that $\mathcal{N}$ is irreducible as an $N H_{2}^{2}$-module, we may assume that $0 \neq \mathcal{N} \otimes T \subseteq W$, for some $T \in \mathrm{ST}(\lambda(i))$. To conclude that $W=\mathcal{L}_{i}$, it suffices to show the following: for any $T \in \mathrm{ST}(\lambda(i))$, there is a sequence $T_{0}, T_{1}, \ldots$ of standard tableaux which starts at $T$ and ends at the canonical tableau such that $T_{k+1}=s_{i_{k}} T_{k}$ for some $i_{k}$. Let $S$ be a standard tableau and suppose that $1,2, \ldots, a-1$ are on the first row, $a, a+1, \ldots, b$ are on the second row and $b+1$ is on the first row again. Then we swap $b$ and $b+1$. 
Repeating the procedure, we reach the stage $a=b$, and when we swap $b$ and $b+1$, we obtain a standard tableau such that $1,2, \ldots, a$ are on the first row and $a+1$ is on the second row. Hence, repeated use of the procedure starting with $T$ gives the desired sequence of standard tableaux.

In the same manner, we may prove that $\widetilde{\mathcal{L}}_{\ell}$ and $E_{i} \mathcal{L}_{j}$, for $j=i \pm 1$, are irreducible. In particular, we have proved (1).

Suppose that $0 \leq i, j \leq \ell-1$. By the definition of the module structure (4.2.2), we have

$$
\operatorname{ch}_{q} \mathcal{L}_{i}=\left(1+q^{2}\right) \sum_{T \in \operatorname{ST}(\lambda(i))} \operatorname{res}(T) .
$$

Thus, $\operatorname{ch}_{q}\left(E_{j} \mathcal{L}_{i}\right)=\operatorname{ch}_{q}\left(E_{i} \mathcal{L}_{j}\right) \neq 0$ if $j=i \pm 1$ and $\operatorname{ch}_{q}\left(E_{j} \mathcal{L}_{i}\right)=0$ otherwise. Since $E_{i} \mathcal{L}_{j}$ are irreducible if they are nonzero, (2) follows. The proof of (3) is similar.

It is clear from the definition of the modules that $E_{\ell-1} \mathcal{L}_{\ell} \simeq E_{\ell} \mathcal{L}_{\ell-1}$. Applying the exact functor $E_{\ell-1}$ to the exact sequence from Corollary 4.3, we obtain the exact sequence

$$
0 \longrightarrow E_{\ell-1} \widetilde{\mathcal{L}}_{\ell} \longrightarrow E_{\ell} \mathcal{L}_{\ell-1} \longrightarrow E_{\ell-1} \widetilde{\mathcal{L}}_{\ell} \longrightarrow 0 .
$$

It is nonsplit because $E_{\ell} \mathcal{L}_{\ell-1}$ is not homogeneous while $E_{\ell-1} \widetilde{\mathcal{L}}_{\ell}$ is homogeneous.

Remark 4.5. The irreducible modules $\mathcal{L}_{0}, \mathcal{L}_{1}, \ldots, \mathcal{L}_{\ell-1}, \widetilde{\mathcal{L}}_{\ell}$ correspond to the Young walls $Y_{0}, Y_{1}, \ldots, Y_{\ell-1}, Y_{\ell}$ given in (1.2.1) via the crystal isomorphism from Theorem 2.8. Hence, some parts of Lemma 4.4 may be proved by using properties (1.2.2) of the Young walls $Y_{i}$.

Proposition 4.6. The irreducible $R^{\Lambda_{0}}\left(2 \delta-\alpha_{i}\right)$-module is unique, for all $i \in I$, and we have the following structure theorem.

(1) For $0 \leq i \leq \ell-1$, we have an algebra isomorphism $R^{\Lambda_{0}}\left(2 \delta-\alpha_{i}\right) \simeq$ $\operatorname{Mat}\left(\operatorname{dim} \mathcal{L}_{i}, \overline{\mathbf{k}}\right)$, where $\mathcal{L}_{i}$ is the unique irreducible $R^{\Lambda_{0}}\left(2 \delta-\alpha_{i}\right)$-module defined in (4.2.2) and

$$
\operatorname{dim} \mathcal{L}_{i}=2\left(\begin{array}{c}
h-2 \\
i
\end{array}\right)-2\left(\begin{array}{c}
h-2 \\
i-1
\end{array}\right) .
$$

(2) We have an algebra isomorphism $R^{\Lambda_{0}}\left(2 \delta-\alpha_{\ell}\right) \simeq \operatorname{Mat}\left(\operatorname{dim} \widetilde{\mathcal{L}}_{\ell}, \mathbf{k}[x] /\left(x^{2}\right)\right)$, where $\widetilde{\mathcal{L}}_{\ell}$ is the unique irreducible $R^{\Lambda_{0}}\left(2 \delta-\alpha_{\ell}\right)$-module defined in Corollary 4.3 and

$$
\operatorname{dim} \widetilde{\mathcal{L}}_{\ell}=\left(\begin{array}{c}
h-2 \\
\ell
\end{array}\right)-\left(\begin{array}{c}
h-2 \\
\ell-1
\end{array}\right) .
$$

Furthermore, $\mathcal{L}_{\ell}$ is the unique indecomposable projective $R^{\Lambda_{0}}\left(2 \delta-\alpha_{\ell}\right)$ module.

Proof. It follows from Lemma 1.2 and Proposition 2.5 that $R^{\Lambda_{0}}\left(2 \delta-\alpha_{i}\right)$ is a simple algebra, for $0 \leq i \leq \ell-1$. Then $\mathcal{L}_{i}$ is the unique irreducible $R^{\Lambda_{0}}\left(2 \delta-\alpha_{i}\right)$-module by Lemma 4.4(1). Thus, $R^{\Lambda_{0}}\left(2 \delta-\alpha_{i}\right) \simeq \operatorname{End}_{\mathbf{k}}\left(\mathcal{L}_{i}\right)$ and the hook length formula for the number of elements in $\operatorname{ST}(\lambda(i))$ gives the formula for $\operatorname{dim} \mathcal{L}_{i}$. Hence (1) follows. To prove (2), observe that

$$
r_{\ell-1} \cdots r_{1} r_{0}\left(\Lambda_{0}-\delta\right)=r_{\ell-1} \cdots r_{1} r_{0}\left(\Lambda_{0}\right)-\delta=\Lambda_{0}-2 \delta+\alpha_{\ell} .
$$

Then, $R^{\Lambda_{0}}\left(2 \delta-\alpha_{\ell}\right)$ and $R^{\Lambda_{0}}(\delta)$ have the same number of simple modules by Proposition 2.5. As $R^{\Lambda_{0}}(\delta) \simeq \mathbf{k}[x] /\left(x^{2}\right), \widetilde{\mathcal{L}}_{\ell}$ is the unique $R^{\Lambda_{0}}\left(2 \delta-\alpha_{\ell}\right)$-module by 
Lemma 4.4(1) again. Let $P_{\ell}$ be the indecomposable projective $R^{\Lambda_{0}}\left(2 \delta-\alpha_{\ell}\right)$-module. Since

$$
\operatorname{dim} R^{\Lambda_{0}}\left(2 \delta-\alpha_{\ell}\right)=\left(\operatorname{dim} P_{\ell}\right)\left(\operatorname{dim} \widetilde{\mathcal{L}}_{\ell}\right)
$$

and Theorem 3.2 shows that $\operatorname{dim} R^{\Lambda_{0}}\left(2 \delta-\alpha_{\ell}\right)=2\left(\operatorname{dim} \widetilde{\mathcal{L}}_{\ell}\right)^{2}, P_{\ell}$ is the uniserial module of length 2. As Corollary 4.3 shows that we have a surjective homomorphism $P_{\ell} \rightarrow \mathcal{L}_{\ell}$, we have $P_{\ell} \simeq \mathcal{L}_{\ell}$. Hence $\mathcal{L}_{\ell}$ is the unique indecomposable projective $R^{\Lambda_{0}}\left(2 \delta-\alpha_{\ell}\right)$-module.

To finish the proof, we observe that the regular representation gives an algebra isomorphism

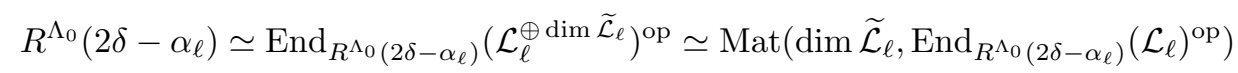

and $\operatorname{End}_{R^{\Lambda_{0}}\left(2 \delta-\alpha_{\ell}\right)}\left(\mathcal{L}_{\ell}\right) \simeq \mathbf{k}[x] /\left(x^{2}\right)$. Thus, $R^{\Lambda_{0}}\left(2 \delta-\alpha_{\ell}\right) \simeq \operatorname{Mat}\left(\operatorname{dim} \widetilde{\mathcal{L}}_{\ell}, \mathbf{k}[x] /\left(x^{2}\right)\right)$.

4.3. Representations of $R^{\Lambda_{0}}(2 \delta)$. In this section, we give explicit constructions of various $R^{\Lambda_{0}}(2 \delta)$-modules using results from Section 4.2 ,

Lemma 4.7. By declaring that $x_{h}$ and $\psi_{h-1}$ act as 0 , and $e(\nu)$, for $\nu \in I^{2 \delta}$, as

$$
e(\nu)(v \otimes T)= \begin{cases}v \otimes T & \text { if } \nu=\operatorname{res}(T) * i, \\ 0 & \text { otherwise, }\end{cases}
$$

where $\operatorname{res}(T) * i$ is the concatenation of $\operatorname{res}(T)$ and $(i)$, the irreducible $R^{\Lambda_{0}}\left(2 \delta-\alpha_{i}\right)$ module $\mathcal{L}_{i}$ extends to an irreducible $R^{\Lambda_{0}}(2 \delta)$-module, for $0 \leq i \leq \ell-1$.

Proof. We may assume that $\nu=\operatorname{res}(T) * i$. Then (4.2.1) tells us that

$$
\nu_{h-2} \neq i=\nu_{h} \quad \text { and } \quad \nu_{h-1}=i \pm 1 \neq \nu_{h} .
$$

Thus, the relations for

$$
\left(\psi_{h-1} \psi_{h-2} \psi_{h-1}-\psi_{h-2} \psi_{h-1} \psi_{h-2}\right) e(\nu) \text { and }\left(\psi_{h-1} x_{l}-x_{s_{k}(l)} \psi_{h-1}\right) e(\nu)
$$

hold. If $\nu_{h-1} \neq \ell$, then $a_{\nu_{h-1}, \nu_{h}}=a_{i \pm 1, i} \neq 0$ and $x_{h-1}(v \otimes T)=0$ imply that

$$
\mathcal{Q}_{\nu_{h-1}, \nu_{h}}\left(x_{h-1}, x_{h}\right)(v \otimes T)=0 \text {. }
$$

If $\nu_{h-1}=\ell$, then $a_{\nu_{h-1}, \nu_{h}} \neq 0$ forces $\nu_{h}=\ell-1$, and $\mathcal{Q}_{\nu_{h-1}, \nu_{h}}\left(x_{h-1}, x_{h}\right)(v \otimes T)$ is equal to

$$
\mathcal{Q}_{\ell, \ell-1}\left(x_{h-1}, x_{h}\right)(v \otimes T)=\left(x_{h-1}^{2}+x_{h}\right)(v \otimes T)=\xi_{2}^{2} v \otimes T=0 .
$$

Thus, the relation for $\psi_{h-1}^{2} e(\nu)$ holds. It is easy to check the remaining defining relations.

Definition 4.8. We denote the irreducible $R^{\Lambda_{0}}(2 \delta)$-module defined in Lemma 4.7 by $\mathcal{S}_{i}$, for $i=0,1, \ldots, \ell-1$.

Lemma 4.9. By declaring that $\psi_{h-1}$ act as $0, x_{h}$ and $e(\nu)$, for $\nu \in I^{2 \delta}$, as

$$
x_{h}(v \otimes T)=\xi_{2} v \otimes T, \quad e(\nu)(v \otimes T)= \begin{cases}v \otimes T & \text { if } \nu=\operatorname{res}(T) * \ell, \\ 0 & \text { otherwise, }\end{cases}
$$

$\mathcal{L}_{\ell}$ extends to an $R^{\Lambda_{0}}(2 \delta)$-module. Further, the $R^{\Lambda_{0}}\left(2 \delta-\alpha_{\ell}\right)$-submodule $\widetilde{\mathcal{L}}_{\ell}$ is stable under the action of $x_{h}$ and $e(\nu)$, for $\nu \in I^{2 \delta}$. 
Proof. We let $\nu=\operatorname{res}(T) * \ell$ and we check the defining relations. By (4.2.1), we have

$$
\nu_{h-2}=\ell-2 \text { or } \ell \quad \text { and } \quad \nu_{h-1}=\ell-1 \neq \ell=\nu_{h} .
$$

Then, it is easy to check the defining relations except for

$$
\left(\psi_{h-1} \psi_{h-2} \psi_{h-1}-\psi_{h-2} \psi_{h-1} \psi_{h-2}\right) e(\nu)
$$

when $\left(\nu_{h-2}, \nu_{h-1}, \nu_{h}\right)=(\ell, \ell-1, \ell)$. In this case, we have to prove

$$
\frac{\mathcal{Q}_{\ell, \ell-1}\left(x_{h-2}, x_{h-1}\right)-\mathcal{Q}_{\ell, \ell-1}\left(x_{h}, x_{h-1}\right)}{x_{h-2}-x_{h}}(v \otimes T)=0 .
$$

But the left hand side is equal to

$$
\left(x_{h-2}+x_{h}\right)(v \otimes T)=\left(\xi_{1}+\xi_{2}\right) v \otimes T=0 .
$$

Hence, we have checked all the defining relations. Finally, it is clear that $\widetilde{\mathcal{L}}_{\ell}$ is stable under the action of $x_{h}$ and $e(\nu)$, for $\nu \in I^{2 \delta}$.

Definition 4.10. We denote the $R^{\Lambda_{0}}(2 \delta)$-module defined in Lemma 4.9 by $\widehat{\mathcal{S}}_{\ell}$ and the unique irreducible $R^{\Lambda_{0}}(2 \delta)$-submodule by $\mathcal{S}_{\ell}$.

By construction, $\varepsilon_{j}\left(\mathcal{S}_{i}\right)=\delta_{i j}$, for $0 \leq i, j \leq \ell$. Hence $\mathcal{S}_{0}, \ldots, \mathcal{S}_{\ell}$ are pairwise nonisomorphic $R^{\Lambda_{0}}(2 \delta)$-modules. Using Theorem 2.8, (1.2.3) and Corollary 4.3, we have the following lemma.

Lemma 4.11. (1) The modules $\mathcal{S}_{i}(i=0,1, \ldots, \ell)$ form a complete list of irreducible $R^{\Lambda_{0}}(2 \delta)$-modules.

(2) There is a nonsplit exact sequence

$$
0 \longrightarrow \mathcal{S}_{\ell} \longrightarrow \widehat{\mathcal{S}}_{\ell} \longrightarrow \mathcal{S}_{\ell} \longrightarrow 0 \text {. }
$$

(3) For $i, j \in I$, we have

$$
E_{j} \mathcal{S}_{i} \simeq \begin{cases}\mathcal{L}_{i} & \text { if } i=j \neq \ell, \\ \widetilde{\mathcal{L}}_{\ell} & \text { if } i=j=\ell, \\ 0 & \text { otherwise. }\end{cases}
$$

To determine the basic algebra of $R^{\Lambda_{0}}(2 \delta)$ and its representation type, we will need a uniserial module $\mathcal{M}$ with $\operatorname{Top}(\mathcal{M}) \simeq \mathcal{S}_{\ell-1}$ and $\operatorname{Rad}(\mathcal{M}) \simeq \widehat{\mathcal{S}}_{\ell}$, which we shall construct here.

Definition 4.12. For each $T \in \mathrm{ST}(\lambda(\ell-1))$ with $\operatorname{res}_{h-1}(T)=\ell$, define $T^{\vee} \in$ $\mathrm{ST}(\lambda(\ell))$ to be the standard tableau obtained from $T$ by moving the node $(1, \ell+2)$ to the node $(2, \ell+1)$.

Example 4.13. The following is an example when $\ell=3$ :
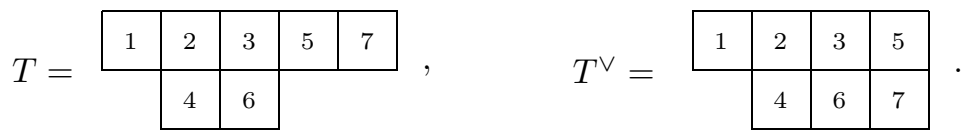

Lemma 4.14. We change the action of $\psi_{h-1}$ on the $R^{\Lambda_{0}}(2 \delta)$-module $\widehat{\mathcal{S}}_{\ell} \oplus \mathcal{S}_{\ell-1}$ to

$$
\psi_{h-1}(v \otimes T)= \begin{cases}v \otimes T^{\vee} & \text { if } \operatorname{res}_{h-1}(T)=\ell, \\ 0 & \text { otherwise, }\end{cases}
$$

and keep the action of the other generators unchanged. Then, we obtain an $R^{\Lambda_{0}}(2 \delta)$ module. 
Proof. It suffices to check the defining relations which involve $\psi_{h-1}$. It also suffices to check the relations on $v \otimes T$ with $T \in \mathrm{ST}(\lambda(\ell-1))$. Note that $\operatorname{res}_{h}(T)=\ell-1$.

Suppose that $\nu \neq \operatorname{res}(T)$. If $e\left(s_{h-1} \nu\right) \psi_{h-1}(v \otimes T) \neq 0$, then we have

$$
\operatorname{res}_{h-1}(T)=\ell, \quad\left(\nu_{h}, \nu_{h-1}\right)=\left(\operatorname{res}_{h-1}\left(T^{\vee}\right), \operatorname{res}_{h}\left(T^{\vee}\right)\right)=(\ell-1, \ell)
$$

and $\nu_{k}=\operatorname{res}_{k}\left(T^{\vee}\right)=\operatorname{res}_{k}(T)$, for $1 \leq k \leq h-2$. It contradicts $\nu \neq \operatorname{res}(T)$, and we have

$$
\psi_{h-1} e(\nu)(v \otimes T)=e\left(s_{h-1} \nu\right) \psi_{h-1}(v \otimes T) .
$$

If $\nu=\operatorname{res}(T)$, then explicit computation for the cases $\operatorname{res}_{h-1}(T) \neq \ell$ and $\operatorname{res}_{h-1}(T)=$ $\ell$ proves $\psi_{h-1} e(\nu)(v \otimes T)=e\left(s_{h-1} \nu\right) \psi_{h-1}(v \otimes T)$.

To prove the remaining defining relations, we may assume that $\nu=\operatorname{res}(T)$.

Suppose that $1 \leq k \leq h-3$. Then the location of $h-1$ in $s_{k} T$ is the same as the location in $T$. It follows that if $\psi_{h-1} \psi_{k}(v \otimes T) \neq 0$ or $\psi_{k} \psi_{h-1}(v \otimes T) \neq 0$, then $\nu_{h-1}=\ell$ and $s_{k} T$ is standard. Hence we assume that $\nu_{h-1}=\ell$ and $s_{k} T$ is standard. Then explicit computation shows $\psi_{h-1} \psi_{k}(v \otimes T)=\psi_{k} \psi_{h-1}(v \otimes T)$.

Next we consider the relation for $\left(\psi_{h-1} x_{k}-x_{s_{h-1}(k)} \psi_{h-1}\right) e(\nu)$. Suppose that $k \neq h-1, h$. If $\psi_{h-1} x_{k}(v \otimes T) \neq 0$ or $x_{k} \psi_{h-1}(v \otimes T) \neq 0$, then $k$ is located at $(1, \ell+1)$ and $h-1$ is located at $(1, \ell+2)$. Hence we assume that $k$ is located at $(1, \ell+1)$ and $h-1$ is located at $(1, \ell+2)$. Then direct computation shows that

$$
\left(\psi_{h-1} x_{k}-x_{k} \psi_{h-1}\right)(v \otimes T)=\xi_{1} v \otimes T^{\vee}-\xi_{1} v \otimes T^{\vee}=0 .
$$

If $k=h$, then $\left(\psi_{h-1} x_{h}-x_{h-1} \psi_{h-1}\right)(v \otimes T)=-x_{h-1} \psi_{h-1}(v \otimes T)=0$. Suppose that $k=h-1$. If $\psi_{h-1} x_{h-1}(v \otimes T) \neq 0$ or $x_{h} \psi_{h-1}(v \otimes T) \neq 0$, then $h-1$ is located at $(1, \ell+2)$. Thus we assume that $h-1$ is located at $(1, \ell+2)$. Then, recalling the action of $x_{h}$ on $\widehat{\mathcal{S}}_{\ell}$, we have

$$
\left(\psi_{h-1} x_{h-1}-x_{h} \psi_{h-1}\right)(v \otimes T)=\xi_{2} v \otimes T^{\vee}-\xi_{2} v \otimes T^{\vee}=0 .
$$

Hence the relation for $\left(\psi_{h-1} x_{k}-x_{s_{h-1}(k)} \psi_{h-1}\right) e(\nu)$ holds.

Since $\psi_{h-1}$ acts as 0 on $\widehat{\mathcal{S}}_{\ell}$, we have $\psi_{h-1}^{2}(v \otimes T)=0$. On the other hand, we already know that

$$
\psi_{h-1}^{2}(v \otimes T)=\mathcal{Q}_{\nu_{h-1}, \nu_{h}}\left(x_{h-1}, x_{h}\right)(v \otimes T)
$$

holds if we replace $\psi_{h-1}$ with 0 , because it is one of the defining relations on $\mathcal{S}_{\ell-1}$. It implies the relation for $\psi_{h-1}^{2} e(\nu)$.

Finally, we check the relation for $\left(\psi_{h-1} \psi_{h-2} \psi_{h-1}-\psi_{h-2} \psi_{h-1} \psi_{h-2}\right) e(\nu)$. Note that $\psi_{h-2}$ acts as 0 on $\widehat{\mathcal{S}}_{\ell}$. Hence $\left(\psi_{h-1} \psi_{h-2} \psi_{h-1}-\psi_{h-2} \psi_{h-1} \psi_{h-2}\right)(v \otimes T)=0$. On the other hand, we have $\nu_{h-2}=\ell-2$ or $\ell$, and $\nu_{h}=\ell-1$. Hence $\nu_{h-2}=\nu_{h}$ does not occur, and the relation for $\left(\psi_{h-1} \psi_{h-2} \psi_{h-1}-\psi_{h-2} \psi_{h-1} \psi_{h-2}\right) e(\nu)$ holds.

Lemma 4.15. Let $\mathcal{M}$ be the $R^{\Lambda_{0}}(2 \delta)$-module constructed in Lemma 4.14. Then the radical series of $\mathcal{M}$ is given as follows:

$$
\mathcal{M} \simeq \begin{aligned}
& \mathcal{S}_{\ell-1} \\
& \mathcal{S}_{\ell} \\
& \mathcal{S}_{\ell} .
\end{aligned}
$$

Proof. Since the action of $\psi_{h-1}$ on $\mathcal{M}$ is nonzero, we have a nonsplit exact sequence

$$
0 \longrightarrow \widehat{\mathcal{S}}_{\ell} \longrightarrow \mathcal{M} \longrightarrow \mathcal{S}_{\ell-1} \longrightarrow 0 .
$$


Hence $\operatorname{Soc}(\mathcal{M}) \subseteq \widehat{\mathcal{S}}_{\ell}$, and $\operatorname{Soc}(\mathcal{M})=\mathcal{S}_{\ell}$ by Lemma 4.11(2). If $\mathcal{S}_{\ell}$ were to appear in $\operatorname{Top}(\mathcal{M})$, then $\mathcal{M} / \operatorname{Soc}(\mathcal{M})$ would be a semisimple module $\mathcal{S}_{\ell-1} \oplus \mathcal{S}_{\ell}$. Then, $\psi_{h-1}$ would act as 0 on $\mathcal{M} / \operatorname{Soc}(\mathcal{M})$. Recall the definiton of the action:

$$
\psi_{h-1}(v \otimes T)= \begin{cases}v \otimes T^{\vee} & \text { if } \operatorname{res}_{h-1}(T)=\ell, \\ 0 & \text { otherwise. }\end{cases}
$$

It follows that the action of $\psi_{h-1}$ on $\mathcal{M} / \operatorname{Soc}(\mathcal{M})$ is also nonzero. Thus, $\operatorname{Top}(\mathcal{M})=$ $\mathcal{S}_{\ell-1}$ and $\operatorname{Rad}(\mathcal{M})=\widehat{\mathcal{S}}_{\ell}$. Then Lemma 4.11 $(2)$ implies that $\operatorname{Rad}^{2}(\mathcal{M})=\operatorname{Soc}(\mathcal{M})$.

\section{REPRESEntation TYPE}

In this section, we give a criterion for the representation type of $R^{\Lambda_{0}}(\beta)$. When $\ell=1$, the algebra $R^{\Lambda_{0}}(\beta)$ is of type $A_{1}^{(1)}$. The Erdmann-Nakano theorem [9] and the isomorphism theorem by Brundan-Kleshchev [4] and Rouquier [28] and [3] for arbitrary parameters tell us that $R^{\Lambda_{0}}(\beta)$ is

(1) simple if $\Lambda_{0}-\beta \in \mathrm{W} \Lambda_{0}$,

(2) not semisimple but of finite type if $\Lambda_{0}-\beta \in \mathrm{W} \Lambda_{0}-\delta$,

(3) of tame type if $\Lambda_{0}-\beta \in \mathrm{W} \Lambda_{0}-2 \delta$,

(4) of wild type otherwise.

Thus, an interesting question is to find a criterion for the representation type of $R^{\Lambda_{0}}(\beta)$ when $\ell \geq 2$. From now on, we assume that $R^{\Lambda_{0}}(\beta)$ is of type $D_{\ell+1}^{(2)}$ for $\ell \geq 2$.

5.1. The algebra $R^{\Lambda_{0}}(\delta)$. In Section 4.1 we showed that $R^{\Lambda_{0}}(\delta)$ is generated by $x_{\ell+1}$ and the algebra homomorphism $\mathbf{k}[x] \rightarrow R^{\Lambda_{0}}(\delta)$ which sends $x$ to $x_{\ell+1}$ induces $\mathbf{k}[x] /\left(x^{2}\right) \simeq R^{\Lambda_{0}}(\delta)$. Thus, $R^{\Lambda_{0}}(\delta)$ is not semisimple but of finite representation type.

5.2. The algebra $R^{\Lambda_{0}}(2 \delta)$. In this subsection, we show that $R^{\Lambda_{0}}(2 \delta)$ is special biserial by computing the radical series of indecomposable projective $R^{\Lambda_{0}}(2 \delta)$ modules.

Recall the $R^{\Lambda_{0}}\left(2 \delta-\alpha_{i}\right)$-modules $\mathcal{L}_{i}$ and $\widetilde{\mathcal{L}}_{\ell}$ defined in Section 4.2 , We define

$$
\mathcal{P}_{i}=F_{i} \mathcal{L}_{i}, \text { for } i=0,1, \ldots, \ell \text {. }
$$

Proposition 4.6 tells that the $R^{\Lambda_{0}}\left(2 \delta-\alpha_{i}\right)$-modules $\mathcal{L}_{i}(0 \leq i \leq \ell)$ are projective. As the functor $F_{i}$ sends projective objects to projective objects, $\mathcal{P}_{i}$ are projective $R^{\Lambda_{0}}(2 \delta)$-modules.

Recall the irreducible $R^{\Lambda_{0}}(2 \delta)$-modules $\mathcal{S}_{i}$ from Lemma 4.11, It follows from Lemma 4.4(1), Lemma 4.11 and the biadjointness of $E_{i}$ and $F_{i}$ that

$$
\begin{aligned}
& \operatorname{Hom}\left(\mathcal{S}_{j}, \mathcal{P}_{i}\right) \simeq \operatorname{Hom}\left(E_{i} \mathcal{S}_{j}, \mathcal{L}_{i}\right) \simeq \begin{cases}\mathbf{k} & \text { if } j=i, \\
0 & \text { if } j \neq i,\end{cases} \\
& \operatorname{Hom}\left(\mathcal{P}_{i}, \mathcal{S}_{j}\right) \simeq \operatorname{Hom}\left(\mathcal{L}_{i}, E_{i} \mathcal{S}_{j}\right) \simeq \begin{cases}\mathbf{k} & \text { if } j=i, \\
0 & \text { if } j \neq i .\end{cases}
\end{aligned}
$$

Thus, $\mathcal{P}_{i}$ is indecomposable and $\operatorname{Top}\left(\mathcal{P}_{i}\right) \simeq \operatorname{Soc}\left(\mathcal{P}_{i}\right) \simeq \mathcal{S}_{i}$. In other words, $\mathcal{P}_{i}$ is the projective cover of $\mathcal{S}_{i}$, for $0 \leq i \leq \ell$, and $R^{\Lambda_{0}}(2 \delta)$ is weakly symmetric. 
Theorem 5.1. The radical series of $\mathcal{P}_{i}$ are given as follows:

$$
\begin{aligned}
& \mathcal{S}_{0} \quad \mathcal{S}_{i} \\
& \mathcal{P}_{0} \simeq \mathcal{S}_{0} \mathcal{S}_{1}, \quad \mathcal{P}_{i} \simeq \mathcal{S}_{i-1} \mathcal{S}_{i+1} \quad(i \neq \ell-1, \ell), \\
& \mathcal{S}_{0} \quad \mathcal{S}_{i} \\
& \mathcal{S}_{\ell-1} \quad \mathcal{S}_{\ell} \\
& \mathcal{P}_{\ell-1} \simeq \mathcal{S}_{\ell-2} \begin{array}{l}
\mathcal{S}_{\ell} \\
\mathcal{S}_{\ell}
\end{array}, \quad \mathcal{P}_{\ell} \simeq \begin{array}{cc}
\mathcal{S}_{\ell} & \mathcal{S}_{\ell-1} \\
\mathcal{S}_{\ell-1} & \mathcal{S}_{\ell}
\end{array} \\
& \mathcal{S}_{\ell-1} \quad \mathcal{S}_{\ell}
\end{aligned}
$$

Proof. We first compute $\operatorname{dim} \operatorname{Hom}\left(\mathcal{P}_{i}, \mathcal{P}_{j}\right)$ to obtain the composition multiplicities.

Suppose that $i \neq j$. By the biadjointness of $E_{i}$ and $F_{i}$ and [15, Thm. 5.1], we have

$$
\operatorname{Hom}\left(\mathcal{P}_{i}, \mathcal{P}_{j}\right) \simeq \operatorname{Hom}\left(E_{j} F_{i} \mathcal{L}_{i}, \mathcal{L}_{j}\right) \simeq \operatorname{Hom}\left(F_{i} E_{j} \mathcal{L}_{i}, \mathcal{L}_{j}\right) \simeq \operatorname{Hom}\left(E_{j} \mathcal{L}_{i}, E_{i} \mathcal{L}_{j}\right),
$$

which yields, by Lemma 4.4

$$
\operatorname{dim} \operatorname{Hom}\left(\mathcal{P}_{i}, \mathcal{P}_{j}\right)= \begin{cases}2 & \text { if }(i, j)=(\ell-1, \ell),(\ell, \ell-1) \\ 1 & \text { if } j=i \pm 1 \text { and }(i, j) \neq(\ell-1, \ell),(\ell, \ell-1) \\ 0 & \text { otherwise }\end{cases}
$$

Next we consider the case $i=j$. As $E_{i} \mathcal{L}_{i}=0$ and $\left\langle h_{i}, \Lambda_{0}-2 \delta+\alpha_{i}\right\rangle>0$, it follows from Theorem 2.6 that

$$
E_{i} F_{i} \mathcal{L}_{i} \simeq \mathcal{L}_{i}^{\oplus\left\langle h_{i}, \Lambda_{0}-2 \delta+\alpha_{i}\right\rangle}
$$

Thus, by Lemma 4.4, we have

$$
\operatorname{dim} \operatorname{Hom}\left(\mathcal{P}_{i}, \mathcal{P}_{i}\right)=\operatorname{dim} \operatorname{Hom}\left(\mathcal{L}_{i}, E_{i} F_{i} \mathcal{L}_{i}\right)= \begin{cases}3 & \text { if } i=0 \\ 2 & \text { if } i=1, \ldots, \ell-1 \\ 4 & \text { if } i=\ell\end{cases}
$$

Therefore, we have

$$
\begin{aligned}
{\left[\mathcal{P}_{0}\right] } & =3\left[\mathcal{S}_{0}\right]+\left[\mathcal{S}_{1}\right] \\
{\left[\mathcal{P}_{i}\right] } & =\left[\mathcal{S}_{i-1}\right]+2\left[\mathcal{S}_{i}\right]+\left[\mathcal{S}_{i+1}\right] \quad(i=1, \ldots, \ell-1), \\
{\left[\mathcal{P}_{\ell-1}\right] } & =\left[\mathcal{S}_{\ell-2}\right]+2\left[\mathcal{S}_{\ell-1}\right]+2\left[\mathcal{S}_{\ell}\right], \\
{\left[\mathcal{P}_{\ell}\right] } & =2\left[\mathcal{S}_{\ell-1}\right]+4\left[\mathcal{S}_{\ell}\right]
\end{aligned}
$$

in the Grothendieck group $K_{0}\left(R^{\Lambda_{0}}(2 \delta)\right.$-mod).

Recall that the algebra $R^{\Lambda_{0}}(\beta)$ has an anti-involution which fixes all the defining generators elementwise, and we have the corresponding duality on the category of $R^{\Lambda_{0}}(\beta)$-modules:

$$
M \mapsto M^{\vee}=\operatorname{Hom}_{\mathbf{k}}(M, \mathbf{k}) .
$$

It is straightforward to check that $\mathcal{S}_{i}$ are self-dual, so that Lemma 4.15 implies that

$$
\mathcal{M} \simeq \begin{gathered}
\mathcal{S}_{\ell-1} \\
\mathcal{S}_{\ell} \\
\mathcal{S}_{\ell}
\end{gathered} \quad \text { and } \quad \mathcal{M}^{\vee} \simeq \begin{gathered}
\mathcal{S}_{\ell} \\
\mathcal{S}_{\ell} \\
\mathcal{S}_{\ell-1}
\end{gathered}
$$


Since $\mathcal{P}_{i}$ is the projective cover of $\mathcal{S}_{i}$, there are nontrivial surjective homomorphisms

$$
\mathcal{P}_{\ell-1} \rightarrow \mathcal{M} \text { and } \quad \mathcal{P}_{\ell} \rightarrow \mathcal{M}^{\vee} \text {. }
$$

As the algebra $R^{\Lambda_{0}}(2 \delta)$ is weakly symmetric, the indecomposable projective module $\mathcal{P}_{i}$ is isomorphic to its dual. Therefore, the heart of $\mathcal{P}_{i}$ is self-dual, which yields the assertion.

Recall that a finite dimensional algebra is a special biserial algebra if the quiver and the relations of its basic algebra satisfy the following conditions.

(a1) For each vertex $i$, the number of incoming arrows is at most 2 .

(a2) For each vertex $i$, the number of outgoing arrows is at most 2 .

(b1) For each arrow $\alpha$, there is at most one arrow $\beta$ such that $\alpha \beta \neq 0$.

(b2) For each arrow $\alpha$, there is at most one arrow $\beta$ such that $\beta \alpha \neq 0$.

The notion of special biserial algebras was originally introduced by Skowroński and Waschbüsch 31 in order to characterize representation-finite algebras which have at most 2 nonprojective indecomposable direct summands in the middle term of any almost split sequence.

If the relations of a special biserial algebra are given by monomials in arrows, the algebra is called a string algebra, and complete classification of indecomposable modules is known [5]. The indecomposable modules are given by string modules and band modules, where strings and bands are certain walks on the double of the quiver. As we will need some computation of string modules and the growth rate for the number of bands, we quickly review necessary materials.

A walk on a quiver is a closed walk if it returns to the initial vertex of the walk. We say that closed walks $w$ and $w^{\prime}$ are cyclically equivalent if $w=w_{1} \cdots w_{n}$, where $w_{i}$ are arrows of the quiver; then $w^{\prime}=w_{i} w_{i+1} \cdots w_{n} w_{1} \cdots w_{i-1}$, for some $i$.

Definition 5.2. Let $\mathrm{k} Q / I$ be a string algebra. For each arrow $\alpha$ in $Q$, we add a new arrow $\alpha^{-1}$ with the opposite direction, and we denote the resulting quiver by $\widehat{Q}$.

(1) A walk $w=w_{1} \cdots w_{n}(n \geq 0)$ on $\widehat{Q}$ is a string if $w_{i+1} \neq w_{i}^{-1}$, for all $i$, and if a subword $w_{i} w_{i+1} \cdots w_{j}$ is a walk on $Q$ or the inverse of a walk on $Q$, then the walk on $Q$ does not vanish in $\mathbf{k} Q / I$. We say that $w$ and $w^{-1}=w_{n}^{-1} \cdots w_{1}^{-1}$ are equivalent strings.

(2) A closed walk $w=w_{1} \cdots w_{n}(n \geq 1)$ on $\widehat{Q}$ is a band if all the powers $w^{m}$ $(m \geq 1)$ are strings and $w$ itself is not cyclically equivalent to a power of a closed walk. If $w^{\prime}$ is cyclically equivalent to $w$ or $w^{-1}$, we say that $w$ and $w^{\prime}$ are equivalent bands.

Remark 5.3. It is more common to call the equivalence classes strings and bands.

For a string $w=w_{1} \cdots w_{n}(n \geq 0)$, we associate an updown diagram. For example, if $w_{1}, w_{2}, w_{3}^{-1}$ and $w_{n}$ are arrows in $Q$, then:

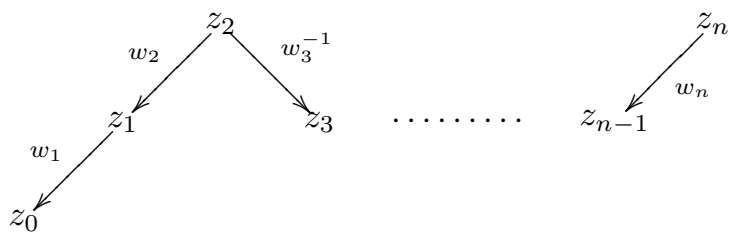


The updown diagram defines the string module $M(w)=\mathbf{k} z_{0} \oplus \cdots \oplus \mathbf{k} z_{n}$. More precisely, the action of an arrow $\alpha$ in $Q$ is defined as follows.

(a) If $w_{i}$ is an arrow in $Q$, then $\alpha z_{i}=\delta_{\alpha, w_{i}} z_{i-1}$.

(b) If $w_{i}^{-1}$ is an arrow in $Q$, then $\alpha z_{i-1}=\delta_{\alpha, w_{i}^{-1}} z_{i}$.

The action of vertices on $z_{i}$ is determined by the end point of $w_{i}$, which is the same as the initial point of $w_{i+1}$, in the obvious manner.

Now, we begin by the following corollary of Theorem 5.1 .

Corollary 5.4. The quiver of $R^{\Lambda_{0}}(2 \delta)$ is given as follows. Moreover, $R^{\Lambda_{0}}(2 \delta)$ is a symmetric special biserial algebra.
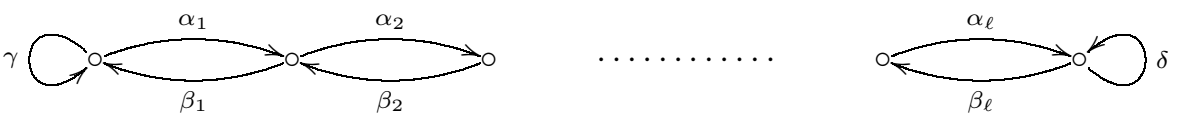

Proof. It follows from Theorem 5.1 that

$$
\operatorname{dim} \operatorname{Hom}\left(\mathcal{P}_{i}, \operatorname{Rad}\left(\mathcal{P}_{j}\right) / \operatorname{Rad}^{2}\left(\mathcal{P}_{j}\right)\right)= \begin{cases}1 & \text { if } j=i \pm 1, \text { or } i=j=0, \ell \\ 0 & \text { otherwise }\end{cases}
$$

We fix lifts of $\operatorname{Hom}\left(\mathcal{P}_{i}, \operatorname{Rad}\left(\mathcal{P}_{j}\right) / \operatorname{Rad}^{2}\left(\mathcal{P}_{j}\right)\right)$ to $\operatorname{Hom}\left(\mathcal{P}_{i}, \mathcal{P}_{j}\right)$ as follows:

$$
\begin{aligned}
\alpha_{i} & =\text { a lift of } \mathcal{P}_{i-1} \rightarrow \mathcal{S}_{i-1} \subseteq \operatorname{Rad}\left(\mathcal{P}_{i}\right) / \operatorname{Rad}^{2}\left(\mathcal{P}_{i}\right), \\
\beta_{i} & =\text { a lift of } \mathcal{P}_{i} \rightarrow \mathcal{S}_{i} \subseteq \operatorname{Rad}\left(\mathcal{P}_{i-1}\right) / \operatorname{Rad}^{2}\left(\mathcal{P}_{i-1}\right), \\
\gamma & =\text { a lift of } \mathcal{P}_{0} \rightarrow \mathcal{S}_{0} \subseteq \operatorname{Rad}\left(\mathcal{P}_{0}\right) / \operatorname{Rad}^{2}\left(\mathcal{P}_{0}\right), \\
\delta & =\text { a lift of } \mathcal{P}_{\ell} \rightarrow \mathcal{S}_{\ell} \subseteq \operatorname{Rad}\left(\mathcal{P}_{\ell}\right) / \operatorname{Rad}^{2}\left(\mathcal{P}_{\ell}\right)
\end{aligned}
$$

Then, $\alpha_{i}, \beta_{i}, \gamma$ and $\delta$ give the quiver of the basic algebra of $R^{\Lambda_{0}}(2 \delta)$, and the relations are

$$
\begin{gathered}
\gamma \alpha_{1}=0, \quad \beta_{1} \gamma=0, \quad \gamma^{2}=\alpha_{1} \beta_{1}, \quad \alpha_{\ell} \beta_{\ell}=0, \quad \delta^{2}=0, \\
\beta_{i} \alpha_{i}=\alpha_{i+1} \beta_{i+1}, \text { for } 1 \leq i \leq \ell-2, \\
\alpha_{i} \alpha_{i+1}=0, \quad \beta_{i+1} \beta_{i}=0, \quad \text { for } 1 \leq i \leq \ell-1, \\
\beta_{\ell-1} \alpha_{\ell-1}=\alpha_{\ell} \delta \beta_{\ell}, \quad \delta \beta_{\ell} \alpha_{\ell}=\beta_{\ell} \alpha_{\ell} \delta .
\end{gathered}
$$

Indeed, considering the configuration of the radical series in Theorem[5.1, $\gamma \alpha_{1}=$ $0, \beta_{1} \gamma=0$ and $\alpha_{i} \alpha_{i+1}=0, \beta_{i+1} \beta_{i}=0$, for $1 \leq i \leq \ell-1$, are clear. As $F_{\ell} \widetilde{\mathcal{L}}_{\ell}$ has composition factors

$$
\left[F_{\ell} \widetilde{\mathcal{L}}_{\ell}\right]=2\left[\mathcal{S}_{\ell}\right]+\left[\mathcal{S}_{\ell-1}\right]
$$

and $\mathcal{P}_{\ell}=F_{\ell} \mathcal{L}_{\ell}$ implies that $\operatorname{Top}\left(F_{\ell} \widetilde{\mathcal{L}}_{\ell}\right) \simeq \operatorname{Top}\left(\mathcal{P}_{\ell}\right)$ and $\operatorname{Soc}\left(F_{\ell} \widetilde{\mathcal{L}}_{\ell}\right) \simeq \operatorname{Soc}\left(\mathcal{P}_{\ell}\right)$,

$$
\begin{aligned}
F_{\ell} \widetilde{\mathcal{L}}_{\ell} \simeq \begin{array}{l}
\mathcal{S}_{\ell} \\
\mathcal{S}_{\ell-1} \\
\mathcal{S}_{\ell},
\end{array}
\end{aligned}
$$

and we have an $R^{\Lambda_{0}}(2 \delta)$-submodule of $\mathcal{P}_{\ell}$ which is isomorphic to $F_{\ell} \widetilde{\mathcal{L}}_{\ell}$. It follows that

$$
\operatorname{Im}(\delta)=\operatorname{Ker}(\delta) \simeq F_{\ell} \widetilde{\mathcal{L}}_{\ell}
$$


and we have $\delta^{2}=0$. Similarly, we have $\operatorname{Im}\left(\alpha_{\ell}\right)=\operatorname{Ker}\left(\beta_{\ell}\right) \simeq \mathcal{M}$ and $\operatorname{Im}\left(\beta_{\ell}\right) \simeq \mathcal{M}^{\vee}$, which implies $\alpha_{\ell} \beta_{\ell}=0$. Further, explicit computation shows that

$$
\begin{aligned}
\operatorname{Im}\left(\gamma^{2}\right) & =\operatorname{Soc}\left(\mathcal{P}_{0}\right)=\operatorname{Im}\left(\alpha_{1} \beta_{1}\right), \\
\operatorname{Im}\left(\beta_{i} \alpha_{i}\right) & =\operatorname{Soc}\left(\mathcal{P}_{i}\right)=\operatorname{Im}\left(\alpha_{i+1} \beta_{i+1}\right), \text { for } 1 \leq i \leq \ell-2, \\
\operatorname{Im}\left(\beta_{\ell-1} \alpha_{\ell-1}\right) & =\operatorname{Soc}\left(\mathcal{P}_{\ell-1}\right)=\operatorname{Im}\left(\alpha_{\ell} \delta \beta_{\ell}\right) .
\end{aligned}
$$

Therefore, $\gamma^{2}, \beta_{i} \alpha_{i}$ and $\beta_{\ell-1} \alpha_{\ell-1}$ are nonzero scalar multiples of $\alpha_{1} \beta_{1}, \alpha_{i+1} \beta_{i+1}$ and $\alpha_{\ell} \delta \beta_{\ell}$, respectively. By adjusting $\alpha_{i}$ 's by nonzero scalar multiples, we may assume that $\gamma^{2}=\alpha_{1} \beta_{1}, \beta_{i} \alpha_{i}=\alpha_{i+1} \beta_{i+1}$ and $\beta_{\ell-1} \alpha_{\ell-1}=\alpha_{\ell} \delta \beta_{\ell}$ hold. To show that $\delta \beta_{\ell} \alpha_{\ell}=\beta_{\ell} \alpha_{\ell} \delta$, it is enough to find that $\operatorname{End}\left(\mathcal{P}_{\ell}\right)$ is commutative. Let $\nu_{0}=$ $(0,1,2, \ldots, \ell) \in I^{\ell+1}$ and $\nu=\nu_{0} * \nu_{0}$. As $e(\nu) \mathcal{S}_{\ell} \neq 0$, we have a surjective map $R^{\Lambda_{0}}(2 \delta) e(\nu) \rightarrow \mathcal{P}_{\ell}$. Thus, $\mathcal{P}_{\ell}$ is a direct summand of $R^{\Lambda_{0}}(2 \delta) e(\nu)$. Then, (5.3.5) below shows that $\operatorname{End}\left(R^{\Lambda_{0}}(2 \delta) e(\nu)\right)$ is a commutative local algebra, and it follows that $\mathcal{P}_{\ell} \simeq R^{\Lambda_{0}}(2 \delta) e(\nu)$ and $\operatorname{End}\left(\mathcal{P}_{\ell}\right)$ is commutative. Hence, all the relations listed above hold, and the dimension consideration proves that they give defining relations of the basic algebra of $R^{\Lambda_{0}}(2 \delta)$. In particular, the quiver of $R^{\Lambda_{0}}(2 \delta)$ is as claimed, and $R^{\Lambda_{0}}(2 \delta)$ is a special biserial algebra.

Next, we construct a trace map on the basic algebra. The basic algebra is $(4 \ell+7)-$ dimensional and the following elements in $B_{1}, \ldots, B_{2 \ell+1}$ altogether form a basis of the basic algebra, which we denote by $B$ :

(1) $B_{1}=\left\{e_{0}, \gamma, \gamma^{2}\right\}$.

(2) $B_{i+1}=\left\{\alpha_{i}, \beta_{i}\right\}$, for $1 \leq i \leq \ell-1$.

(3) $B_{i+\ell}=\left\{e_{i}, \alpha_{i+1} \beta_{i+1}\right\}$, for $1 \leq i \leq \ell-2$.

(4) $B_{2 \ell-1}=\left\{e_{\ell-1}, \alpha_{\ell} \delta \beta_{\ell}\right\}$.

(5) $B_{2 \ell}=\left\{\alpha_{\ell}, \beta_{\ell}, \alpha_{\ell} \delta, \delta \beta_{\ell}\right\}$

(6) $B_{2 \ell+1}=\left\{e_{\ell}, \delta, \beta_{\ell} \alpha_{\ell}, \delta \beta_{\ell} \alpha_{\ell}\right\}$.

The basis $B$ has the property that either $b_{1} b_{2}=0$ or $b_{1} b_{2} \in B$, for $b_{1}, b_{2} \in B$. We define the trace map by values on $B$, and we declare that the nonzero values are

$$
\operatorname{Tr}\left(\gamma^{2}\right)=\operatorname{Tr}\left(\alpha_{2} \beta_{2}\right)=\cdots=\operatorname{Tr}\left(\alpha_{\ell-1} \beta_{\ell-1}\right)=\operatorname{Tr}\left(\alpha_{\ell} \delta \beta_{\ell}\right)=\operatorname{Tr}\left(\delta \beta_{\ell} \alpha_{\ell}\right)=1 .
$$

Note that $\operatorname{Tr}(b) \neq 0$, for $b \in B$, only when the source and the sink of $b$ coincide. We consider the matrix $\left(\operatorname{Tr}\left(b_{1} b_{2}\right)\right)_{b_{1}, b_{2} \in B}$. Our task is to show that it is a nonsingular symmetric matrix. Suppose that the source of $b_{1}$ is different from the sink of $b_{2}$. Then $\operatorname{Tr}\left(b_{1} b_{2}\right)=0$ and $b_{2} b_{1}=0$. If the source of $b_{1}$ equals the sink of $b_{2}$, $\left\{b_{1}, b_{2}\right\} \subseteq B_{i}$, for some $i$. Therefore, it is enough to show that the submatrix $\left(\operatorname{Tr}\left(b_{1} b_{2}\right)\right)_{b_{1}, b_{2} \in B_{i}}$ is a nonsingular symmetric matrix. But it can be checked by direct computation.

We remark that there exists an example of weakly symmetric special biserial algebras which is not symmetric. See [32, IV.2.8].

Corollary 5.5. The algebra $R^{\Lambda_{0}}(2 \delta)$ is of tame type.

Proof. Since biserial algebras have tame or finite representation type [6] (for special biserial algebras it was already proved in [33]), we show that $R^{\Lambda_{0}}(2 \delta)$ is not of finite type. But if we set $x=\delta$ and $y=\beta_{\ell} \alpha_{\ell}$, where $\delta, \alpha_{\ell}, \beta_{\ell}$ are as in Corollary 5.4, we have $\operatorname{End}\left(\mathcal{P}_{\ell}\right)=\mathbf{k}[x, y] /\left(x^{2}, y^{2}\right)$. The Kronecker algebra $\mathbf{k}[x, y] /\left(x^{2}, y^{2}\right)$ is of tame type, so that $R^{\Lambda_{0}}(2 \delta)$ is not of finite type. 
Based on [8], 30] and [24, Erdmann and Skowroński have obtained a detailed description of the Auslander-Reiten quiver of a special biserial self-injective algebra [10, Thm. 2.1, Thm. 2.2]. In particular, [10, Thm. 2.2] says that if there are infinitely many equivalence classes of bands, then its stable Auslander-Reiten quiver has finitely many nonhomogeneous tubes $\mathbb{Z} A_{\infty} /\left\langle\tau^{p}\right\rangle$, infinitely many homogeneous tubes, and infinitely many components of the form $\mathbb{Z} A_{\infty}^{\infty}$, where $\tau$ is the AuslanderReiten translation. We may give a more explicit result for $R^{\Lambda_{0}}(2 \delta)$ in type $D_{\ell+1}^{(2)}$ $(\ell \geq 2)$, as follows.

Proposition 5.6. The stable Auslander-Reiten quiver of $R^{\Lambda_{0}}(2 \delta)$ has

(i) the unique nonhomogeneous tube $\mathbb{Z} A_{\infty} /\left\langle\tau^{2 \ell+1}\right\rangle$ consisting of string modules,

(ii) one homogeneous tube consisting of string modules,

(iii) infinitely many homogeneous tubes consisting of band modules,

(iv) infinitely many components of the form $\mathbb{Z} A_{\infty}^{\infty}$ consisting of string modules.

Proof. Let $A=\mathbf{k} Q / I$ be the basic algebra of $R^{\Lambda_{0}}(2 \delta)$ given in Corollary 5.4 and define $B=A / \operatorname{Soc}(A)$. Then $B$ is the string algebra defined by the quiver $Q$ and the relations

$$
\begin{aligned}
& \gamma^{2}=0, \quad \gamma \alpha_{1}=0, \quad \beta_{1} \gamma=0, \\
& \alpha_{i} \beta_{i}=0 \text {, for } 1 \leq i \leq \ell \text {, } \\
& \beta_{i} \alpha_{i}=0, \text { for } 1 \leq i \leq \ell-1 \text {, } \\
& \alpha_{i} \alpha_{i+1}=0 \text {, for } 1 \leq i \leq \ell-1 \text {, } \\
& \beta_{i+1} \beta_{i}=0 \text {, for } 1 \leq i \leq \ell-1 \text {, } \\
& \delta \beta_{\ell} \alpha_{\ell}=\beta_{\ell} \alpha_{\ell} \delta=0, \quad \alpha_{\ell} \delta \beta_{\ell}=0, \quad \delta^{2}=0 .
\end{aligned}
$$

First we show that $B$ has infinitely many equivalence classes of bands. Then it implies that $A$ is not of polynomial growth by the same argument as in [29, Lem. 1], and we are in the case of [10, Thm. 2.2].

We define closed walks $a$ and $b$ as follows. Note that $\beta_{\ell} \alpha_{\ell} \neq 0$ in $B$.

$$
\begin{array}{lrl}
a=\delta \alpha_{\ell}^{-1} \beta_{\ell}^{-1}, & b=\delta \alpha_{\ell}^{-1} \beta_{\ell-1} \cdots \alpha_{2}^{-1} \beta_{1} \gamma^{-1} \alpha_{1} \beta_{2}^{-1} \cdots \alpha_{\ell-1} \beta_{\ell}^{-1} & \text { if } \ell \text { is even. } \\
a=\delta^{-1} \beta_{\ell} \alpha_{\ell}, \quad b=\delta^{-1} \beta_{\ell} \alpha_{\ell-1}^{-1} \cdots \alpha_{2}^{-1} \beta_{1} \gamma^{-1} \alpha_{1} \beta_{2}^{-1} \cdots \alpha_{\ell} & \text { if } \ell \text { is odd. }
\end{array}
$$

We claim that $2^{q}-2$ closed walks $\left\{x_{1} \cdots x_{q} \mid x_{i}=a\right.$ or $\left.b\right\} \backslash\left\{a^{q}, b^{q}\right\}$, for a prime $q$, give $\left(2^{q}-2\right) / q$ equivalence classes of bands. It is clear that their powers are strings. Thus, it suffices to show that it is not cyclically equivalent to a power of its subword. Suppose that this is the case. Then, we may assume that the subword starts with $\delta$ or $\delta^{-1}$. But $\delta^{ \pm}$appear only as the first alphabet of $a$ and $b$, so that the subword is of the form $x_{i} x_{i+1} \cdots x_{i+r-1}$ and $r$ divides $q$. Thus, $r=1$ or $r=q$ because $q$ is a prime, and $r=1$ does not occur because we have excluded $a^{q}$ and $b^{q}$. We have proved that $x_{1} \cdots x_{q}$ cannot be cyclically equivalent to a power of its subword, and we have the claim. In particular, we have constructed infinitely many equivalence classes of bands. Since all the nonprojective indecomposable $A$-modules are indecomposable $B$-modules and the stable Auslander-Reiten quiver of $A$ coincides with the Auslander-Reiten quiver of $B$, we consider the latter. Then, by the general result [10, Thm. 2.2], it only remains to determine the period of string $B$-modules in components of the form $\mathbb{Z} A_{\infty} /\left\langle\tau^{p}\right\rangle$, for $p \geq 1$, since band $B$-modules belong to 
homogeneous tubes [5, p. 165]. But string modules on the boundary of such components are known [5, p. 170]. They are $\left\{B e_{i} / B \alpha \mid i\right.$ is the end point of an arrow $\left.\alpha\right\}$. Thus, it suffices to determine the period of these string modules with respect to $\tau=D \operatorname{Tr}$.

First of all, $B e_{\ell} / B \delta$ is exceptional and it has period 1. Indeed,

$$
B e_{\ell} \rightarrow B e_{\ell} \rightarrow B e_{\ell} / B \delta=\frac{\left\langle e_{\ell}, \delta, \alpha_{\ell}, \beta_{\ell} \alpha_{\ell}, \alpha_{\ell} \delta\right\rangle_{\mathbf{k}}}{\left\langle\delta, \alpha_{\ell} \delta\right\rangle_{\mathbf{k}}}=M\left(\beta_{\ell} \alpha_{\ell}\right)
$$

where $B e_{\ell} \rightarrow B e_{\ell}$ is given by $x \mapsto x \delta$, is the projective resolution, and direct computation shows that $\tau\left(M\left(\beta_{\ell} \alpha_{\ell}\right)\right) \simeq M\left(\beta_{\ell} \alpha_{\ell}\right)$.

Similarly, we compute the $\tau$-orbit through $B e_{0} / B \gamma=M\left(\beta_{1}\right)$. Suppose that $\ell$ is even. The first almost split sequence to consider is

$$
0 \rightarrow M\left(\alpha_{1}\right) \rightarrow M\left(\beta_{1} \gamma^{-1} \alpha_{1}\right) \rightarrow M\left(\beta_{1}\right) \rightarrow 0
$$

and we have $\tau\left(B e_{0} / B \gamma\right)=B e_{1} / B \beta_{2}$. The rule to construct almost split sequences for the modules of the form $B e_{i} / B \alpha$ is that if $B e_{i} / B \alpha=M(u)$ and $\tau\left(B e_{i} / B \alpha\right)=$ $M(v)$, then we have the almost split sequence

$$
0 \rightarrow M(v) \rightarrow M\left(u \alpha^{-1} v\right) \rightarrow M(u) \rightarrow 0 .
$$

We proceed further as follows:

$$
\begin{gathered}
\tau\left(B e_{0} / B \gamma\right)=M\left(\alpha_{1}\right)=B e_{1} / B \beta_{2}, \\
\cdots \cdots \\
\tau^{\frac{\ell}{2}}\left(B e_{0} / B \gamma\right)=M\left(\alpha_{\ell-1}\right)=B e_{\ell-1} / B \beta_{\ell}, \\
\tau^{\frac{\ell}{2}+1}\left(B e_{0} / B \gamma\right)=M\left(\delta \beta_{\ell}\right)=B e_{\ell-1} / B \alpha_{\ell-1}, \\
\tau^{\frac{\ell}{2}+2}\left(B e_{0} / B \gamma\right)=M\left(\beta_{\ell-2}\right)=B e_{\ell-3} / B \alpha_{\ell-3}, \\
\cdots \cdots \\
\tau^{\ell}\left(B e_{0} / B \gamma\right)=M\left(\beta_{2}\right)=B e_{1} / B \alpha_{1} .
\end{gathered}
$$

We continue the computation and obtain

$$
\begin{gathered}
\tau^{\ell+1}\left(B e_{0} / B \gamma\right)=M(\gamma)=B e_{0} / B \beta_{1}, \\
\tau^{\ell+2}\left(B e_{0} / B \gamma\right)=M\left(\alpha_{2}\right)=B e_{2} / B \beta_{3}, \\
\ldots \ldots \\
\tau^{\frac{3}{2} \ell}\left(B e_{0} / B \gamma\right)=M\left(\alpha_{\ell-2}\right)=B e_{\ell-2} / B \beta_{\ell-1}, \\
\tau^{\frac{3}{2} \ell+1}\left(B e_{0} / B \gamma\right)=M\left(\alpha_{\ell} \delta\right)=B e_{\ell} / B \alpha_{\ell}, \\
\tau^{\frac{3}{2} \ell+2}\left(B e_{0} / B \gamma\right)=M\left(\beta_{\ell-1}\right)=B e_{\ell-2} / B \alpha_{\ell-2}, \\
\cdots \cdots \\
\tau^{2 \ell}\left(B e_{0} / B \gamma\right)=M\left(\beta_{3}\right)=B e_{2} / B \alpha_{2}, \\
\tau^{2 \ell+1}\left(B e_{0} / B \gamma\right)=M\left(\beta_{1}\right)=B e_{0} / B \gamma .
\end{gathered}
$$


Therefore, the period of $B e_{0} / B \gamma$ is $2 \ell+1$, and the $\tau$-orbit contains all the string modules of the form $B e_{i} / B \alpha$ but $B e_{\ell} / B \delta$. Suppose that $\ell$ is odd. Then the computation is entirely similar and we reach the same conclusion. We have proved that there exist a unique nonhomogeneous tube $\mathbb{Z} A_{\infty} /\left\langle\tau^{2 \ell+1}\right\rangle$ consisting of string modules and a unique homogeneous tube consisting of string modules.

By the main theorem given below, Proposition 5.6 gives the shape of stable Auslander-Reiten quivers for finite quiver Hecke algebras of tame type in type $D_{\ell+1}^{(2)}$.

5.3. The algebra $R^{\Lambda_{0}}(3 \delta)$. In this subsection, we show that $R^{\Lambda_{0}}(3 \delta)$ is of wild type.

Let $\nu_{0}=(0,1,2, \ldots, \ell) \in I^{\ell+1}$ and, for $r=0,1,2$ and $0 \leq s \leq \ell$, or $r=3$ and $s=0$, set

$$
\begin{aligned}
\nu^{r, s} & =\underbrace{\nu_{0} * \cdots * \nu_{0}}_{r} *(0,1,2, \ldots, s-1), \\
\beta^{r, s} & =r \delta+\alpha_{0}+\alpha_{1}+\cdots+\alpha_{s-1},
\end{aligned}
$$

where $\nu * \nu^{\prime}$ is the concatenation of $\nu$ and $\nu^{\prime}$. Note that $\nu^{r, s} \in I^{\beta^{r, s}}$. Using the residue pattern (1.3.1), one can show that there exists only one standard tableau $T$ such that $\operatorname{res}(T)=\nu^{r, s}$. Thus, by Theorem 3.2 , we have

$$
\operatorname{dim} e\left(\nu^{r, s}\right) R^{\Lambda_{0}}\left(\beta^{r, s}\right) e\left(\nu^{r, s}\right)=2^{r} .
$$

Proposition 5.7. The algebra $e\left(\nu^{3,0}\right) R^{\Lambda_{0}}(3 \delta) e\left(\nu^{3,0}\right)$ is isomorphic to the quotient algebra of $\mathbf{k}[x, y, z]$ by the ideal generated by $x^{2}, y^{2}-a x y$ and $z^{2}-b x y-c y z-d y z$ for some $a, b, c, d \in \mathbf{k}$.

Proof. We set $e^{r, s}=e\left(\nu^{r, s}\right)$, for $r=0,1,2$ and $0 \leq s \leq \ell$, or $r=3$ and $s=0$. By a direct computation, we have

$$
\left\langle h_{s}, \Lambda_{0}-\beta^{r, s}\right\rangle= \begin{cases}1 & \text { if } s=0, \ldots, \ell-1 \\ 2 & \text { if } s=\ell\end{cases}
$$

Thus, Theorem 2.6 shows that there are $\left(R^{\Lambda_{0}}\left(\beta^{r, s}\right), R^{\Lambda_{0}}\left(\beta^{r, s}\right)\right)$-bimodule monomorphisms

$$
\begin{aligned}
& R^{\Lambda_{0}}\left(\beta^{r, s}\right) \hookrightarrow e\left(\beta^{r, s}, s\right) R^{\Lambda_{0}}\left(\beta^{r, s+1}\right) e\left(\beta^{r, s}, s\right) \quad(0 \leq s \leq \ell-1) \\
& \bigoplus_{k=0}^{1} R^{\Lambda_{0}}\left(\beta^{r, \ell}\right) \otimes t^{k} \hookrightarrow e\left(\beta^{r, \ell}, \ell\right) R^{\Lambda_{0}}\left(\beta^{r+1,0}\right) e\left(\beta^{r, \ell}, \ell\right) \quad(s=\ell),
\end{aligned}
$$

which yield $\left(e^{r, s} R^{\Lambda_{0}}\left(\beta^{r, s}\right) e^{r, s}, e^{r, s} R^{\Lambda_{0}}\left(\beta^{r, s}\right) e^{r, s}\right)$-bimodule monomorphisms

$$
\begin{aligned}
& e^{r, s} R^{\Lambda_{0}}\left(\beta^{r, s}\right) e^{r, s} \hookrightarrow e^{r, s+1} R^{\Lambda_{0}}\left(\beta^{r, s+1}\right) e^{r, s+1}(0 \leq s \leq \ell-1), \\
& \bigoplus_{k=0}^{1}\left(e^{r, \ell} R^{\Lambda_{0}}\left(\beta^{r, \ell}\right) e^{r, \ell}\right) \otimes t^{k} \hookrightarrow e^{r+1,0} R^{\Lambda_{0}}\left(\beta^{r+1,0}\right) e^{r+1,0} \quad(s=\ell) .
\end{aligned}
$$


We first consider the algebra $e^{1,0} R^{\Lambda_{0}}\left(\beta^{1,0}\right) e^{1,0}$. Since $R^{\Lambda_{0}}\left(\beta^{1,0}\right)=R^{\Lambda_{0}}(\delta)$ and $e\left(\nu^{1,0}\right)$ is the unit element of $R^{\Lambda_{0}}(\delta)$, we have

$$
e^{1,0} R^{\Lambda_{0}}\left(\beta^{1,0}\right) e^{1,0}=R^{\Lambda_{0}}(\delta)=\mathbf{k}[x] /\left(x^{2}\right), \text { for } x=x_{\ell+1} e^{1,0} .
$$

Since $\operatorname{dim} e^{1, s} R^{\Lambda_{0}}\left(\beta^{1, s}\right) e^{1, s}=2$ by (5.3.1), the first monomorphism of (5.3.2) gives

$$
e^{1,0} R^{\Lambda_{0}}\left(\beta^{1,0}\right) e^{1,0} \simeq e^{1,1} R^{\Lambda_{0}}\left(\beta^{1,1}\right) e^{1,1} \simeq \cdots \simeq e^{1, \ell} R^{\Lambda_{0}}\left(\beta^{1, \ell}\right) e^{1, \ell},
$$

and it follows that

$$
e^{1, \ell} R^{\Lambda_{0}}\left(\beta^{1, \ell}\right) e^{1, \ell}=\mathbf{k}[x] /\left(x^{2}\right), \text { for } x=x_{\ell+1} e^{1, \ell} .
$$

Let us consider the second monomorphism of (5.3.2), for $r=1$. It is

$$
\bigoplus_{k=0}^{1}\left(e^{1, \ell} R^{\Lambda_{0}}\left(\beta^{1, \ell}\right) e^{1, \ell}\right) \otimes t^{k} \hookrightarrow e^{2,0} R^{\Lambda_{0}}\left(\beta^{2,0}\right) e^{2,0},
$$

where $f \otimes t^{k}$, for $f \in e^{1, \ell} R^{\Lambda_{0}}\left(\beta^{1, \ell}\right) e^{1, \ell}$, maps to $f x_{2 \ell+2}^{k} e^{2,0}$. Note that it is not only a bimodule homomorphism but an algebra homomorphism since $x_{\ell+1}$ commutes with $x_{2 \ell+2}$ and the monomorphism is induced by the algebra homomorphism

$$
e^{1, \ell} R^{\Lambda_{0}}\left(\beta^{1, \ell}\right) e^{1, \ell} \otimes \mathbf{k}[t] \longrightarrow e^{2,0} R^{\Lambda_{0}}\left(\beta^{2,0}\right) e^{2,0} .
$$

It follows from

$$
2 \operatorname{dim} e^{1, \ell} R^{\Lambda_{0}}\left(\beta^{1, \ell}\right) e^{1, \ell}=4=\operatorname{dim} e^{2,0} R^{\Lambda_{0}}\left(\beta^{2,0}\right) e^{2,0}
$$

that the embedding (5.3.4) is an isomorphism of algebras. Therefore, using (5.3.3) and the fact that $e^{2,0} R^{\Lambda_{0}}\left(\beta^{2,0}\right) e^{2,0}$ is graded, we can conclude

$$
e^{2,0} R^{\Lambda_{0}}\left(\beta^{2,0}\right) e^{2,0}=\mathbf{k}[x, y] /\left(x^{2}, y^{2}-a x y\right)
$$

for some $a \in \mathbf{k}$, where $x=x_{\ell+1} e^{2,0}$ and $y=x_{2 \ell+2} e^{2,0}$.

In the same manner, as $\operatorname{dim} e^{2, s} R^{\Lambda_{0}}\left(\beta^{2, s}\right) e^{2, s}=4$ by (5.3.1), the first monomorphism of (5.3.2) gives

$$
e^{2,0} R^{\Lambda_{0}}\left(\beta^{2,0}\right) e^{2,0} \simeq e^{2,1} R^{\Lambda_{0}}\left(\beta^{2,1}\right) e^{2,1} \simeq \cdots \simeq e^{2, \ell} R^{\Lambda_{0}}\left(\beta^{2, \ell}\right) e^{2, \ell},
$$

which implies

$$
e^{2, \ell} R^{\Lambda_{0}}\left(\beta^{2, \ell}\right) e^{2, \ell} \simeq \mathbf{k}[x, y] /\left(x^{2}, y^{2}-a x y\right) .
$$

Then, because of the dimension equality

$$
2 \operatorname{dim} e^{2, \ell} R^{\Lambda_{0}}\left(\beta^{2, \ell}\right) e^{2, \ell}=8=\operatorname{dim} e^{3,0} R^{\Lambda_{0}}\left(\beta^{3,0}\right) e^{3,0},
$$

the second monomorphism of (5.3.2),

$$
\bigoplus_{k=0}^{1}\left(e^{2, \ell} R^{\Lambda_{0}}\left(\beta^{2, \ell}\right) e^{2, \ell}\right) \otimes t^{k} \hookrightarrow e^{3,0} R^{\Lambda_{0}}\left(\beta^{3,0}\right) e^{3,0},
$$

sending $f \otimes t^{k}$ to $f x_{3 \ell+3}^{k} e^{3,0}\left(f \in e^{2, \ell} R^{\Lambda_{0}}\left(\beta^{2, \ell}\right) e^{2, \ell}\right)$ is an isomorphism of algebras. Therefore, the assertion follows from (5.3.6) and the fact that $e^{3,0} R^{\Lambda_{0}}\left(\beta^{3,0}\right) e^{3,0}$ is graded. 
Corollary 5.8. The algebra $R^{\Lambda_{0}}(3 \delta)$ is of wild type.

Proof. Let $A=e\left(\nu^{3,0}\right) R^{\Lambda_{0}}(3 \delta) e\left(\nu^{3,0}\right)$. It is enough to show that $A$ is wild. By Proposition 5.7 there is a surjective homomorphism

$$
A \rightarrow \mathbf{k}[x, y, z] /\left(x^{2}, y^{2}, z^{2}, x y, y z, z x\right) .
$$

Since the algebra $\mathbf{k}[x, y, z] /\left(x^{2}, y^{2}, z^{2}, x y, y z, z x\right)$ is wild [27, (1.2)], so is $A$.

5.4. Representation type of $R^{\Lambda_{0}}(\beta)$. In this subsection, we show our main theorem, which shows the representation type of $R^{\Lambda_{0}}(\beta)$.

Lemma 5.9 ([9, Prop. 2.3]). Let $A$ and $B$ be finite dimensional $\mathbf{k}$-algebras and suppose that there exists a constant $C>0$ and functors

$$
F: A-\bmod \rightarrow B \text {-mod, } G: B \text {-mod } \rightarrow A \text {-mod }
$$

such that, for any $A$-module $M$,

(1) $M$ is a direct summand of $G F(M)$ as an A-module,

(2) $\operatorname{dim} F(M) \leq C \operatorname{dim} M$.

Then, if $A$ is wild, so is $B$.

Remark 5.10. In [9, Prop. 2.3], the authors use lengths of the modules to state the result. But they use dimensions of the modules in the proof. Let us quickly review their proof. We prove that if $B$ is tame, then $A$ is weakly tame. That is, we show that there are finitely many $(\mathbf{k}[T], A)$-bimodules such that, for any indecomposable $A$-module $M$ with $\operatorname{dim} M \leq d, M$ is a direct summand of one of the bimodules tensored with $\mathbf{k}[T] /(T-\lambda)$, for some $\lambda \in \mathbf{k}$. As $B$ is assumed to be tame, we have finitely many $(\mathbf{k}[T], B)$-bimodules such that every indecomposable $B$-module with dimension at most $C d$ is one of the bimodules tensored with $\mathbf{k}[T] /(T-\lambda)$. Thus, if $\operatorname{dim} F(M)$ is bounded above by $C d$, there are finitely many $(\mathbf{k}[T], B)$-bimodules such that $F(M)$ is a direct summand of one of them tensored with $\mathbf{k}[T] /(T-\lambda)$. By applying the functor $G$ to the bimodules, we obtain finitely many $(\mathbf{k}[T], A)$ bimodules with the required property. The reason the authors use lengths is that we can replace $A$ or $B$ with Morita equivalent algebras freely if we use lengths, and it is good for their purposes.

Proposition 5.11. The algebras $R^{\Lambda_{0}}(k \delta)(k \geq 3)$ are wild.

Proof. For $k \in \mathbb{Z}_{\geq 0}$ and $0 \leq i \leq \ell$, we have

$$
\left\langle h_{i}, \Lambda_{0}-k \delta-\alpha_{0}-\cdots-\alpha_{i-1}\right\rangle= \begin{cases}1 & \text { if } i=0, \ldots, \ell-1, \\ 2 & \text { if } i=\ell .\end{cases}
$$

Thus, the functor $F_{i}: R^{\Lambda_{0}}\left(k \delta+\alpha_{0}+\cdots+\alpha_{i-1}\right)-\bmod \rightarrow R^{\Lambda_{0}}\left(k \delta+\alpha_{0}+\cdots+\alpha_{i}\right)$-mod satisfies the assumptions of Lemma 5.9 by Proposition 2.3 and Theorem 2.6. Hence, if $R^{\Lambda_{0}}\left(k \delta+\alpha_{0}+\cdots+\alpha_{i-1}\right)$ is wild, so is $R^{\Lambda_{0}}\left(k \delta+\alpha_{0}+\cdots+\alpha_{i}\right)$. As $R^{\Lambda_{0}}(3 \delta)$ is wild by Corollary [5.8, the assertion follows by induction.

Recall that a weight $\mu$ with $V\left(\Lambda_{0}\right)_{\mu} \neq 0$ can be written as

$$
\mu=\kappa-k \delta
$$

for some $\kappa \in \mathrm{W} \Lambda_{0}$ and $k \in \mathbb{Z}_{\geq 0}$ and a weight $\mu$ of the above form always satisfies $V\left(\Lambda_{0}\right)_{\mu} \neq 0$ [13, (12.6.1)]. Note that the pair $(\kappa, k)$ is determined uniquely by $\mu$. Then our main theorem, Theorem 5.12 below, follows from Proposition 2.5. Corollary 5.5 and Proposition 5.11. 
Theorem 5.12. For $\kappa \in \mathrm{W} \Lambda_{0}$ and $k \in \mathbb{Z}_{\geq 0}$, the finite quiver Hecke algebra $R^{\Lambda_{0}}\left(\Lambda_{0}-\kappa+k \delta\right)$ of type $D_{\ell+1}^{(2)}(\ell \geq 2)$ is

(1) simple if $k=0$,

(2) of finite representation type but not semisimple if $k=1$,

(3) of tame representation type if $k=2$,

(4) of wild representation type if $k \geq 3$.

We give two remarks. Firstly, if $k=1$, then $R^{\Lambda_{0}}\left(\Lambda_{0}-\kappa+\delta\right)$ is a matrix algebra over $\mathbf{k}[x] /\left(x^{2}\right)$. To prove this, observe that $R^{\Lambda_{0}}(\delta)=\mathbf{k}[x] /\left(x^{2}\right)$ is the Brauer tree algebra with one edge and no exceptional vertex. Then [25, Thm. 4.2] implies that $R^{\Lambda_{0}}\left(\Lambda_{0}-\kappa+\delta\right)$ is Morita equivalent to the Brauer tree algebra with one edge and no exceptional vertex, which is $\mathbf{k}[x] /\left(x^{2}\right)$. Thus, if we denote the dimension of the unique irreducible $R^{\Lambda_{0}}\left(\Lambda_{0}-\kappa+\delta\right)$-module by $d$, we have $R^{\Lambda_{0}}\left(\Lambda_{0}-\kappa+\delta\right) \simeq \operatorname{Mat}\left(d, \mathbf{k}[x] /\left(x^{2}\right)\right)$.

Secondly, if $k=2$, then $R^{\Lambda_{0}}\left(\Lambda_{0}-\kappa+2 \delta\right)$ is a symmetric algebra by [26, Cor. 5.3]. If the results in [1] are correct, then we may conclude that $R^{\Lambda_{0}}\left(\Lambda_{0}-\kappa+2 \delta\right)$ is a symmetric special biserial algebra. Note that the main results in [23] are incorrect, as is mentioned in [3, Example A.7]. Further, $R^{\Lambda_{0}}\left(\Lambda_{0}-\kappa+2 \delta\right)$ has the same stable Auslander-Reiten quiver as $R^{\Lambda_{0}}(2 \delta)$, which is given in Proposition 5.6.

To summarize, if $R^{\Lambda_{0}}(\beta)$ is not of wild representation type, then we know more than its representation type. For example, knowing the stable Auslander-Reiten quiver implies that we may label indecomposable modules in some sense.

\section{ACKNOWLEDGEMENT}

The authors are grateful to Professor Skowroński for his advice on special biserial algebras. Using his results [10] with Professor Erdmann in particular, the authors were able to determine the shape of the stable Auslander-Reiten quiver of tame finite quiver Hecke algebras mentioned in the introduction.

\section{RefERENCES}

[1] T. Adachi, T. Aihara, and A. Chan, Tilting Brauer graph algebras I: Classification of twoterm tilting complexes, arXiv:1504.04827 (2015).

[2] Susumu Ariki and Euiyong Park, Representation type of finite quiver Hecke algebras of type $A_{2 \ell}^{(2)}$, J. Algebra 397 (2014), 457-488, DOI 10.1016/j.jalgebra.2013.09.005. MR 3119233

[3] S. Ariki, K. Iijima, and E. Park, Representation type of finite quiver Hecke algebras of type $A_{\ell}^{(1)}$ for arbitrary parameters, arXiv:1311.4677, to appear in Int. Math. Res. Not. (doi:10.1093/imrn/rnu115).

[4] Jonathan Brundan and Alexander Kleshchev, Blocks of cyclotomic Hecke algebras and Khovanov-Lauda algebras, Invent. Math. 178 (2009), no. 3, 451-484, DOI 10.1007/s00222009-0204-8. MR2551762 (2010k:20010)

[5] M. C. R. Butler and Claus Michael Ringel, Auslander-Reiten sequences with few middle terms and applications to string algebras, Comm. Algebra 15 (1987), no. 1-2, 145-179, DOI 10.1080/00927878708823416. MR876976 (88a:16055)

[6] William Crawley-Boevey, Tameness of biserial algebras, Arch. Math. (Basel) 65 (1995), no. 5, 399-407, DOI 10.1007/BF01198070. MR.1354686 (96i:16021)

[7] Etsurō Date, Michio Jimbo, Masaki Kashiwara, and Tetsuji Miwa, Transformation groups for soliton equations. Euclidean Lie algebras and reduction of the KP hierarchy, Publ. Res. Inst. Math. Sci. 18 (1982), no. 3, 1077-1110, DOI 10.2977/prims/1195183297. MR688946 (84m:58119g)

[8] Piotr Dowbor and Andrzej Skowroński, Galois coverings of representation-infinite algebras, Comment. Math. Helv. 62 (1987), no. 2, 311-337, DOI 10.1007/BF02564450. MR896100 (88m:16020) 
[9] Karin Erdmann and Daniel K. Nakano, Representation type of Hecke algebras of type A, Trans. Amer. Math. Soc. 354 (2002), no. 1, 275-285 (electronic), DOI 10.1090/S0002-994701-02848-3. MR 1859276 (2002j:20011)

[10] Karin Erdmann and Andrzej Skowroński, On Auslander-Reiten components of blocks and self-injective biserial algebras, Trans. Amer. Math. Soc. 330 (1992), no. 1, 165-189, DOI 10.2307/2154159. MR1144759 (93b:16022)

[11] Jin Hong and Seok-Jin Kang, Introduction to quantum groups and crystal bases, Graduate Studies in Mathematics, vol. 42, American Mathematical Society, Providence, RI, 2002. MR:1881971 (2002m:17012)

[12] Michio Jimbo and Tetsuji Miwa, Solitons and infinite-dimensional Lie algebras, Publ. Res. Inst. Math. Sci. 19 (1983), no. 3, 943-1001, DOI 10.2977/prims/1195182017. MR723457 (85i:58060)

[13] Victor G. Kac, Infinite-dimensional Lie algebras, 3rd ed., Cambridge University Press, Cambridge, 1990. MR:1104219 (92k:17038)

[14] Seok-Jin Kang, Crystal bases for quantum affine algebras and combinatorics of Young walls, Proc. London Math. Soc. (3) 86 (2003), no. 1, 29-69, DOI 10.1112/S0024611502013734. MR.1971463 (2004c:17028)

[15] Seok-Jin Kang and Masaki Kashiwara, Categorification of highest weight modules via Khovanov-Lauda-Rouquier algebras, Invent. Math. 190 (2012), no. 3, 699-742, DOI 10.1007/s00222-012-0388-1. MR2995184

[16] M. Kashiwara, On crystal bases of the Q-analogue of universal enveloping algebras, Duke Math. J. 63 (1991), no. 2, 465-516, DOI 10.1215/S0012-7094-91-06321-0. MR.1115118 (93b:17045)

[17] Masaki Kashiwara, The crystal base and Littelmann's refined Demazure character formula, Duke Math. J. 71 (1993), no. 3, 839-858, DOI 10.1215/S0012-7094-93-07131-1. MR.1240605 (95b:17019)

[18] Masaki Kashiwara, Biadjointness in cyclotomic Khovanov-Lauda-Rouquier algebras, Publ. Res. Inst. Math. Sci. 48 (2012), no. 3, 501-524, DOI 10.2977/PRIMS/78. MR2973390

[19] Mikhail Khovanov and Aaron D. Lauda, A diagrammatic approach to categorification of quantum groups. I, Represent. Theory 13 (2009), 309-347, DOI 10.1090/S1088-4165-09-00346-X. MR2525917 (2010i:17023)

[20] Mikhail Khovanov and Aaron D. Lauda, A diagrammatic approach to categorification of quantum groups II, Trans. Amer. Math. Soc. 363 (2011), no. 5, 2685-2700, DOI 10.1090/S00029947-2010-05210-9. MR2763732 (2012a:17021)

[21] Aaron D. Lauda, An introduction to diagrammatic algebra and categorified quantum $\mathfrak{s l}_{2}$, Bull. Inst. Math. Acad. Sin. (N.S.) 7 (2012), no. 2, 165-270. MR3024893

[22] Aaron D. Lauda and Monica Vazirani, Crystals from categorified quantum groups, Adv. Math. 228 (2011), no. 2, 803-861, DOI 10.1016/j.aim.2011.06.009. MR2822211 (2012i:17013)

[23] Zygmunt Pogorzały, Algebras stably equivalent to self-injective special biserial algebras, Comm. Algebra 22 (1994), no. 4, 1127-1160, DOI 10.1080/00927879408824898. MR.1261252 (95b:16008)

[24] Zygmunt Pogorzały and Andrzej Skowroński, Self-injective biserial standard algebras, J. Algebra 138 (1991), no. 2, 491-504, DOI 10.1016/0021-8693(91)90183-9. MR1102821 (92f:16012)

[25] Jeremy Rickard, Derived categories and stable equivalence, J. Pure Appl. Algebra 61 (1989), no. 3, 303-317, DOI 10.1016/0022-4049(89)90081-9. MR.1027750 (91a:16004)

[26] Jeremy Rickard, Derived equivalences as derived functors, J. London Math. Soc. (2) 43 (1991), no. 1, 37-48, DOI 10.1112/jlms/s2-43.1.37. MR.1099084 (92b:16043)

[27] Claus Michael Ringel, The representation type of local algebras, Proceedings of the International Conference on Representations of Algebras (Carleton Univ., Ottawa, Ont., 1974), Carleton Math. Lecture Notes, No. 9, Carleton Univ., Ottawa, Ont., 1974, 24 pp. MR0382356 (52 \#3241)

[28] R. Rouquier, 2 Kac-Moody algebras, arXiv:0812.5023 (2008).

[29] Andrzej Skowroński, Group algebras of polynomial growth, Manuscripta Math. 59 (1987), no. 4, 499-516, DOI 10.1007/BF01170851. MR916000 (88m:16035)

[30] Andrzej Skowroński, Selfinjective algebras of polynomial growth, Math. Ann. 285 (1989), no. 2, 177-199, DOI 10.1007/BF01443513. MR1016089 (90k:16024)

[31] Andrzej Skowroński and Josef Waschbüsch, Representation-finite biserial algebras, J. Reine Angew. Math. 345 (1983), 172-181. MR717892 (85e:16051) 
[32] Andrzej Skowroński and Kunio Yamagata, Frobenius algebras. I, Basic representation theory, EMS Textbooks in Mathematics, European Mathematical Society (EMS), Zürich, 2011. MR2894798

[33] Burkhard Wald and Josef Waschbüsch, Tame biserial algebras, J. Algebra 95 (1985), no. 2, 480-500, DOI 10.1016/0021-8693(85)90119-X. MR801283 (87a:16056)

Department of Pure and Applied Mathematics, Graduate School of Information Science and Technology, Osaka University, Toyonaka, Osaka 560-0043, Japan

E-mail address: ariki@ist.osaka-u.ac.jp

Department of Mathematics, University of Seoul, Seoul 130-743, Republic of Korea

E-mail address: epark@uos.ac.kr 